\title{
Wind Turbine Generator System Power Performance Test Report for the ARE442 Wind Turbine
}

Technical Report NREL/TP-500-46191

February 2010

Jeroen van Dam and Dave Jager

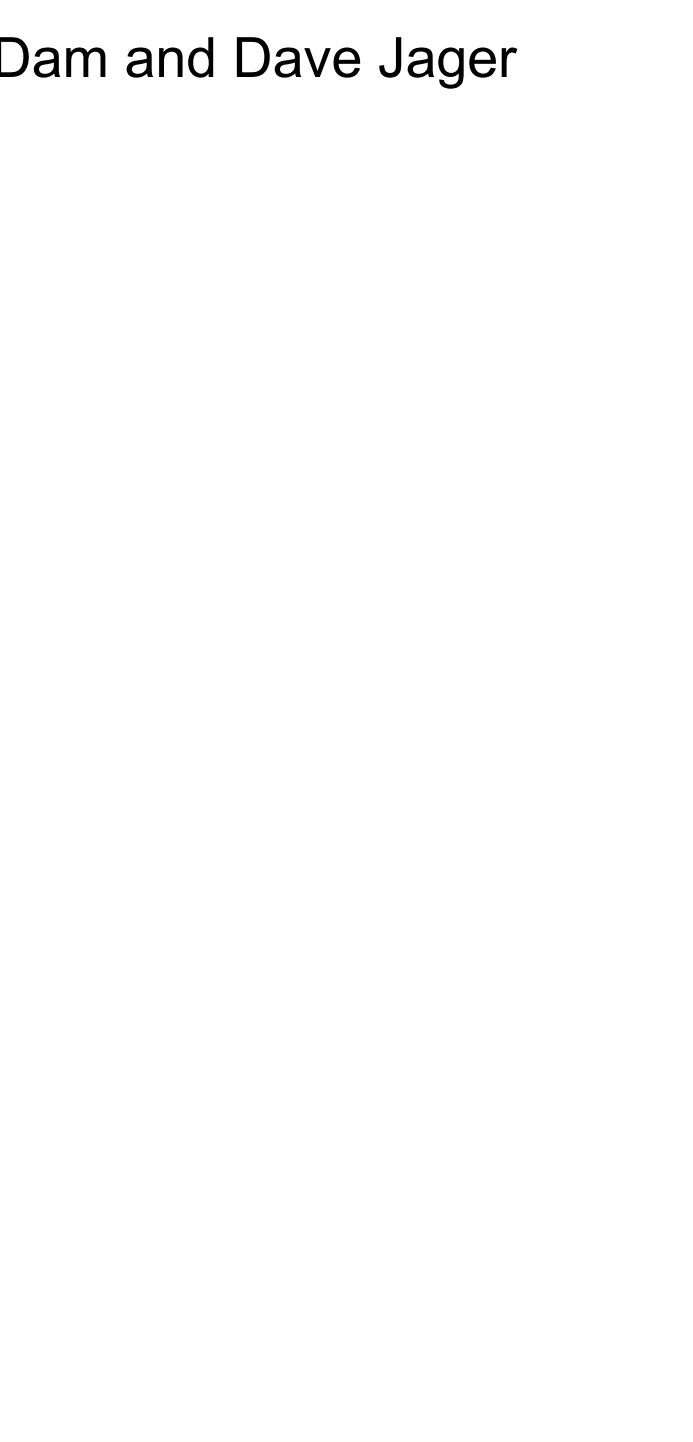




\section{Wind Turbine Generator System Power Performance Test Report for the ARE442 Wind Turbine}

Jeroen van Dam and Dave Jager

Prepared under Task No. WE102211

Technical Report NREL/TP-500-46191

February 2010

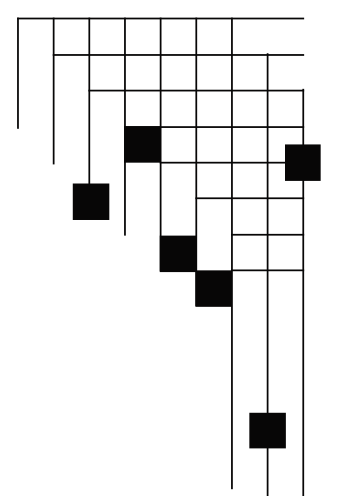




\section{NOTICE}

This report was prepared as an account of work sponsored by an agency of the United States government. Neither the United States government nor any agency thereof, nor any of their employees, makes any warranty, express or implied, or assumes any legal liability or responsibility for the accuracy, completeness, or usefulness of any information, apparatus, product, or process disclosed, or represents that its use would not infringe privately owned rights. Reference herein to any specific commercial product, process, or service by trade name, trademark, manufacturer, or otherwise does not necessarily constitute or imply its endorsement, recommendation, or favoring by the United States government or any agency thereof. The views and opinions of authors expressed herein do not necessarily state or reflect those of the United States government or any agency thereof.

Available electronically at http://www.osti.gov/bridge

Available for a processing fee to U.S. Department of Energy and its contractors, in paper, from:

U.S. Department of Energy

Office of Scientific and Technical Information

P.O. Box 62

Oak Ridge, TN 37831-0062

phone: 865.576 .8401

fax: 865.576 .5728

email: mailto:reports@adonis.osti.gov

Available for sale to the public, in paper, from:

U.S. Department of Commerce

National Technical Information Service

5285 Port Royal Road

Springfield, VA 22161

phone: 800.553.6847

fax: 703.605.6900

email: orders@ntis.fedworld.gov

online ordering: http://www.ntis.gov/ordering.htm 
Notice

This report was prepared by the National Renewable Energy Laboratory (NREL), operated for the United States Department of Energy (DOE) by the Alliance for Sustainable Energy, LLC (Alliance), as an account of work sponsored by the United States government. The test results documented in this report define the characteristics of the test article as configured and under the conditions tested.

THIS REPORT IS PROVIDED "AS IS" AND NEITHER THE GOVERNMENT, ALLIANCE, NREL NOR ANY OF THEIR EMPLOYEES, MAKES ANY WARRANTY, EXPRESS OR IMPLIED, INCLUDING THE WARRANTIES OF MERCHANTABILITY AND FITNESS FOR A PARTICULAR PURPOSE, OR ASSUMES ANY LEGAL LIABILITY OR RESPONSIBILITY FOR THE ACCURACY, COMPLETENESS, OR USEFULNESS OF ANY SUCH INFORMATION DISCLOSED IN THE REPORT, OR OF ANY APPARATUS, PRODUCT, OR PROCESS DISCLOSED, OR REPRESENTS THAT ITS USE WOULD NOT INFRINGE PRIVATELY OWNED RIGHTS.

Neither Alliance nor the U. S. Government shall be liable for special, consequential or incidental damages. Reference herein to any specific commercial product, process, or service by trade name, trademark, manufacturer, or otherwise does not necessarily constitute or imply its endorsement, recommendation, or favoring by the United States government or any agency thereof. The views and opinions of the authors expressed herein do not necessarily state or reflect those of the United States government or any agency thereof or Alliance.

NREL is a DOE Laboratory, and as an adjunct of the United States government, cannot certify wind turbines. The information in this report is limited to NREL's knowledge and understanding as of this date.

NREL is accredited by the American Association for Laboratory Accreditation (A2LA) and the results shown in this test report have been determined in accordance with the NREL's terms of accreditation unless stated otherwise in the report.

This report shall not be reproduced, except in full, without the written approval of Alliance or successor operator of NREL.

Approval By:

Jeroen van Dam, NREL Test Engineer Date

Review By:

Amy Bowen, NREL Test Engineer

Date 


\section{Contents}

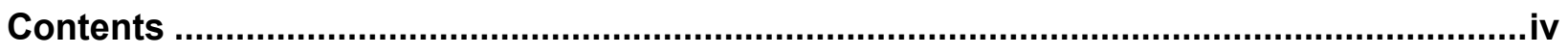

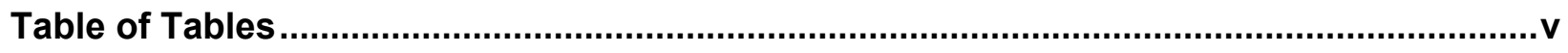

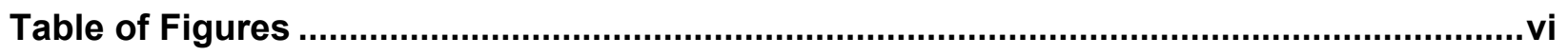

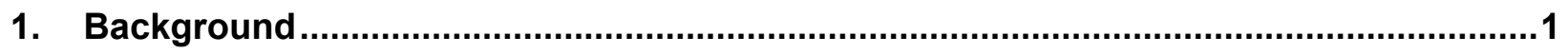

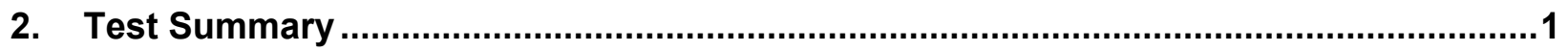

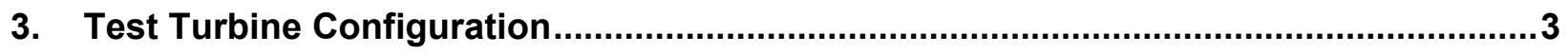

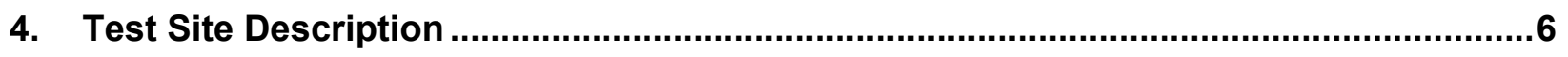

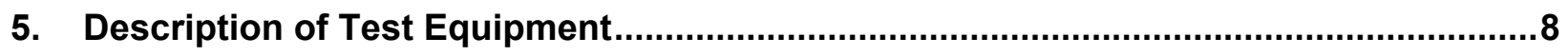

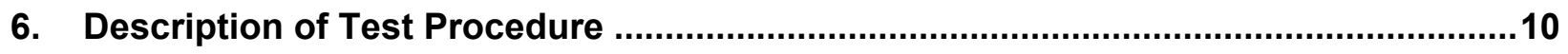

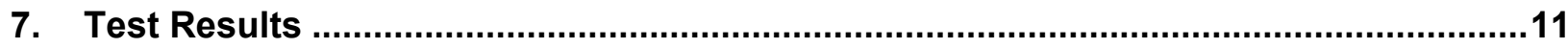

7.1. Tabular Results of Power Performance Test...................................................... 11

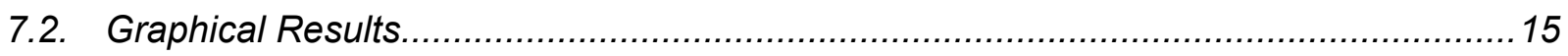

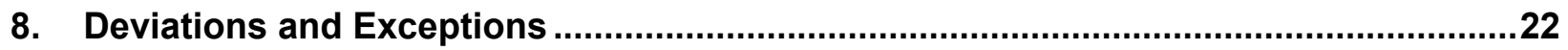

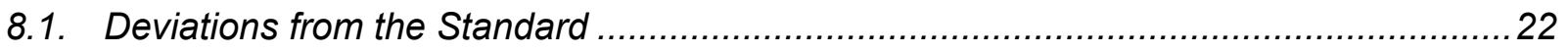

8.2. Exceptions to NWTC-CT Quality Assurance System ...........................................22

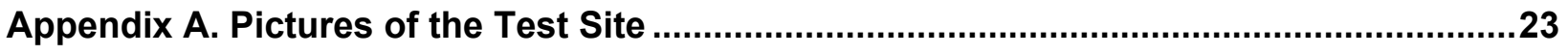

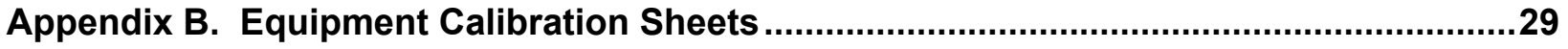




\section{Table of Tables}

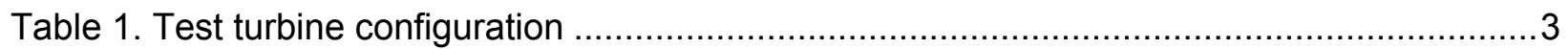

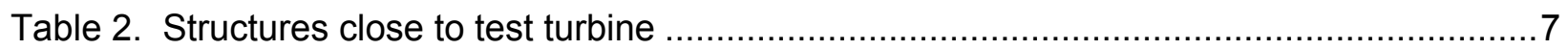

Table 3. Criteria for test site without site calibration ..................................................... 7

Table 4. Equipment used in the power performance test ............................................. 8

Table 5. Uncertainty values used in the analysis ................................................................ 10

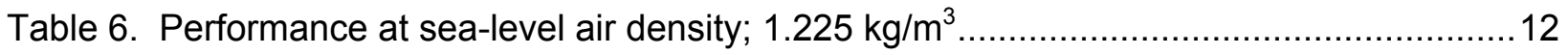

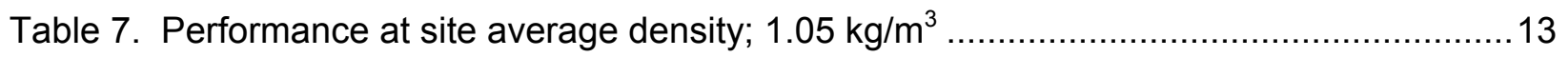

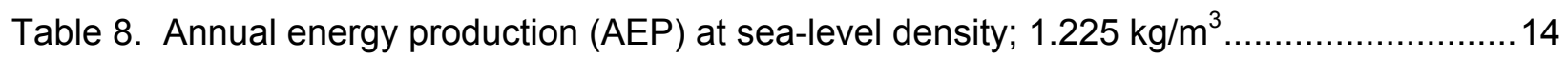

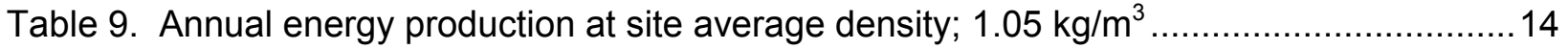




\section{Table of Figures}

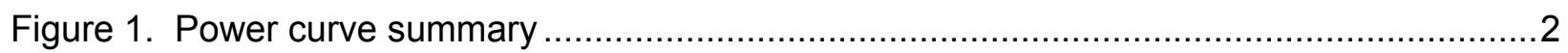

Figure 2. Electrical diagram of the ARE 442 installation ................................................ 4

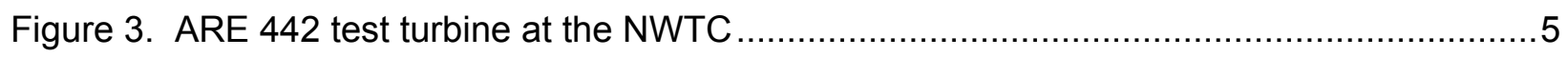

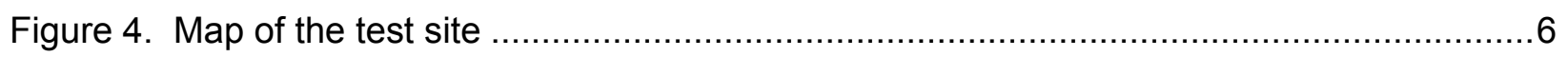

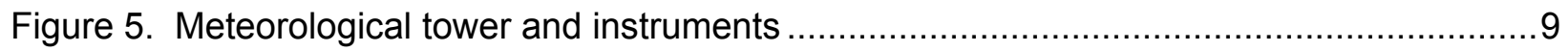

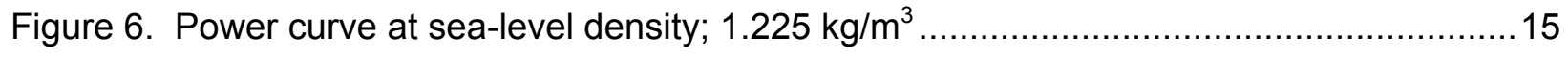

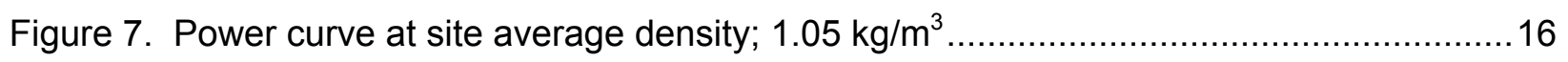

Figure 8. Scatter plot of mean, standard deviation, minimum, and maximum power data. $1 \mathrm{~Hz}$

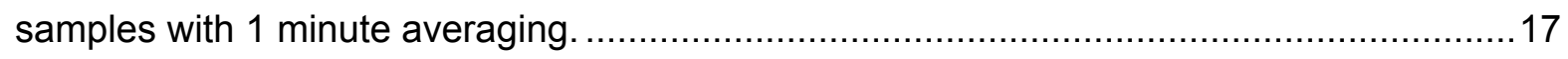

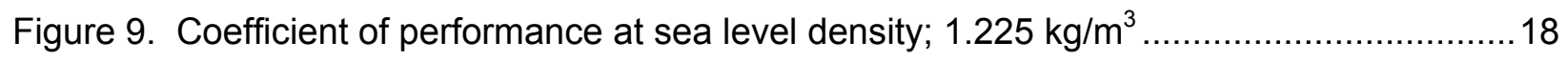

Figure 10. Wind turbulence intensity as a function of wind speed ....................................19

Figure 11. Wind speed and turbulence intensity as a function of wind direction....................20

Figure 12. Rotor speed as a function of wind speed (1-minute averages) and binned values ..21 


\section{Background}

This test is being conducted as part of the U.S. Department of Energy's (DOE) Independent Testing project. This project was established to help reduce the barriers of wind energy expansion by providing independent testing results for small turbines. In total, four turbines are being tested at the National Wind Technology Center (NWTC) as a part of this project. Power performance testing is one of up to 5 tests that may be performed on the turbines, including duration, safety and function, noise, and power quality tests.

\section{Test Summary}

Figure 1 is a summary of the results of a power performance test that NREL conducted on the ARE 442 wind turbine. For this test, the ARE 442 turbine was installed at the NWTC, close to Boulder, Colorado. This test was conducted in accordance with the International Electrotechnical Commission's (IEC) standard, Wind Turbine Generator Systems Part 12: Power Performance Measurements of Electricity Producing Wind Turbines, IEC 61400-12-1 Ed.1.0, 2005-12. However, because the ARE 442 is a small turbine as defined by IEC, NREL also followed Annex $\mathrm{H}$ that applies to small wind turbines. This test report refers to these procedures as the Standard.

In these summary results, wind speed is normalized to sea-level air density. Additional results are given in Section 7. This test was begun on December 9, 2008 and was ended on December 28,2008 . 303.7 hours of valid data were collected during that time. The highest bin filled was the $18.0 \mathrm{~m} / \mathrm{s}$ bin. The amount of test data is sufficient to meet the requirements of the Standard, Annex $\mathrm{H}$. 
Energy Laboratory

Power Performance Test ARE442

Sea-Level Density Power Curve

Report Created:

31-Dec-08

Turbine Specifications:

Serial number:

Rated Power:

Cut-in Wind Speed:

Cut-out Wind Speed:

Rated Wind Speed:

Rotor Diameter:

Control Type:

Pitch Setting:

$\begin{array}{rr}10000 & \mathrm{~W} \\ 2.2 & \mathrm{~m} / \mathrm{s} \\ - & \mathrm{m} / \mathrm{s} \\ 12 & \mathrm{~m} / \mathrm{s} \\ 7.2 & \mathrm{~m} \\ & \\ \text { Active } & \\ \text { Fixed } & \end{array}$

Site Conditions:

Location:

Average Air Density:

Measurement Sectors:

NWTC Boulder, CO

$1.035 \mathrm{~kg} / \mathrm{m}^{\wedge} 3$

214-74 ${ }^{\circ} \mathrm{T}$

Test Statistics:

Start Date:

End Date:

Amount of Data Collected:

Highest Bin Filled:

Test Completed?

9-Dec-2008

28-Dec-2008

303.70 hours

$18.0 \mathrm{~m} / \mathrm{s}$

Yes

\begin{tabular}{|c|c|c|c|}
\hline $\begin{array}{c}\text { Bin Wind } \\
\text { Speed } \\
(\mathrm{m} / \mathrm{s})\end{array}$ & $\begin{array}{c}\text { Bin } \\
\text { Power } \\
\text { (kW) }\end{array}$ & $\begin{array}{c}\text { Number } \\
\text { Data } \\
\text { Points }\end{array}$ & $C p$ \\
\hline 0.51 & -0.03 & 1,000 & -9.00 \\
\hline 1.00 & -0.04 & 1,210 & -1.61 \\
\hline 1.50 & -0.04 & 1,339 & -0.53 \\
\hline 1.99 & -0.05 & 1,423 & -0.27 \\
\hline 2.50 & -0.05 & 1,196 & -0.13 \\
\hline 2.98 & -0.01 & 1,061 & -0.02 \\
\hline 3.49 & 0.08 & 861 & 0.08 \\
\hline 4.00 & 0.30 & 713 & 0.19 \\
\hline 4.49 & 0.59 & 647 & 0.26 \\
\hline 5.00 & 0.91 & 579 & 0.29 \\
\hline 5.51 & 1.35 & 604 & 0.32 \\
\hline 6.00 & 1.86 & 586 & 0.35 \\
\hline 6.50 & 2.42 & 624 & 0.35 \\
\hline 7.00 & 3.04 & 647 & 0.35 \\
\hline 7.49 & 3.70 & 599 & 0.35 \\
\hline 8.00 & 4.41 & 535 & 0.35 \\
\hline 8.49 & 5.19 & 544 & 0.34 \\
\hline 9.00 & 6.02 & 478 & 0.33 \\
\hline 9.50 & 6.81 & 416 & 0.32 \\
\hline 10.00 & 7.70 & 404 & 0.31 \\
\hline 10.49 & 8.47 & 384 & 0.29 \\
\hline 10.99 & 9.17 & 362 & 0.28 \\
\hline 11.50 & 9.69 & 340 & 0.26 \\
\hline 12.01 & 10.10 & 289 & 0.23 \\
\hline 12.49 & 10.05 & 245 & 0.21 \\
\hline 12.97 & 9.91 & 243 & 0.18 \\
\hline 13.50 & 9.42 & 186 & 0.15 \\
\hline 14.00 & 9.00 & 162 & 0.13 \\
\hline 14.48 & 8.58 & 138 & 0.11 \\
\hline 15.00 & 8.28 & 124 & 0.10 \\
\hline 15.54 & 7.86 & 74 & 0.08 \\
\hline 15.99 & 7.85 & 78 & 0.08 \\
\hline 16.49 & 7.59 & 47 & 0.07 \\
\hline 16.95 & 7.33 & 33 & 0.06 \\
\hline 17.51 & 7.48 & 27 & 0.06 \\
\hline 18.01 & 7.57 & 24 & 0.05 \\
\hline
\end{tabular}

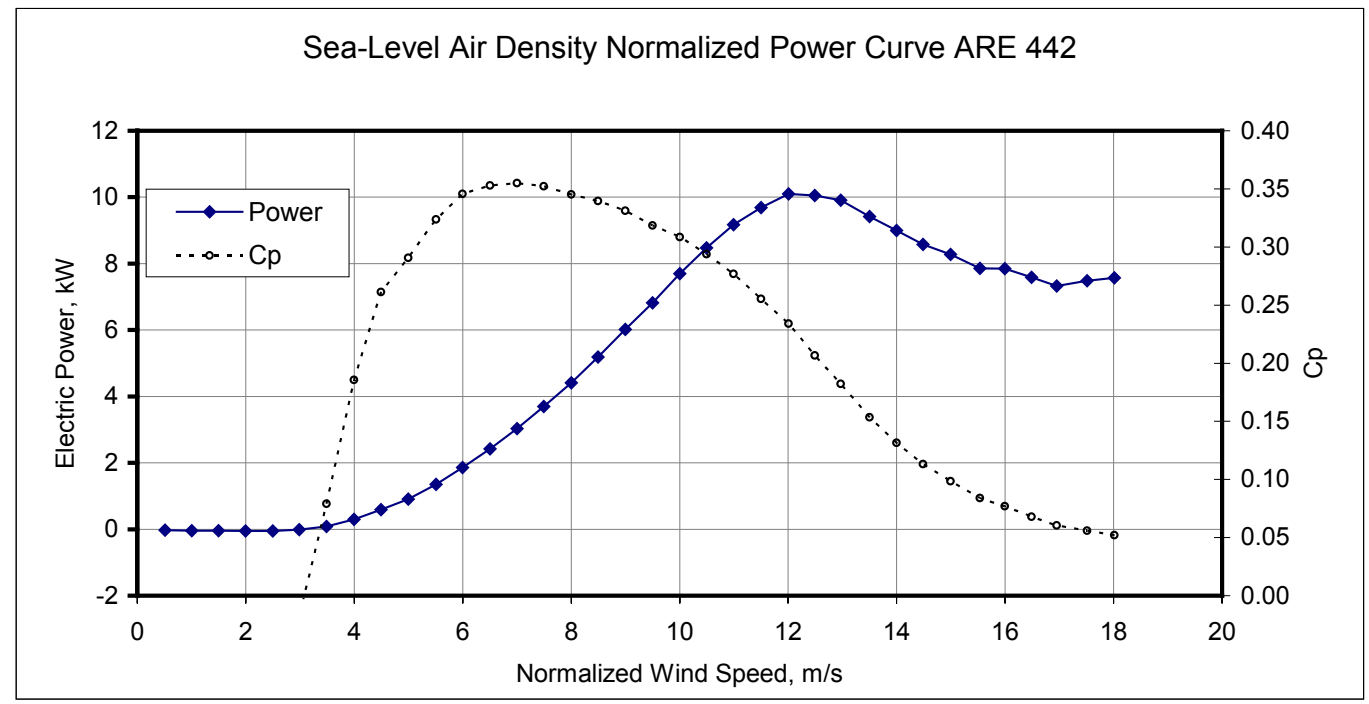

Figure 1. Power curve summary 


\section{Test Turbine Configuration}

Table 1. Test turbine configuration

\begin{tabular}{|c|c|}
\hline $\begin{array}{l}\text { Turbine make, model, serial number, production } \\
\text { year }\end{array}$ & Abundant Renewable Energy, ARE 442, Y08-001C, 2008 \\
\hline Rotor diameter $(\mathrm{m})$ & 7.2 \\
\hline Hub height $(\mathrm{m})$ & 30.9 \\
\hline Tower type & Free standing lattice, Valmont U4.5 x 100' \\
\hline Rated electrical power (kW) & 10 \\
\hline Rated wind speed (m/s) & 12 \\
\hline Rotor speed range (rpm) & $0-140$ \\
\hline Fixed or variable pitch & Fixed \\
\hline Number of blades & 3 \\
\hline Blade-tip pitch angle (deg) & $0^{\circ}$, blade root flat on alternator \\
\hline Blade make, type, serial number & Aero Energy 089-028, 089-029, 089-030 \\
\hline $\begin{array}{l}\text { Description of control system (device \& software } \\
\text { version) }\end{array}$ & $\begin{array}{l}\text { Side furling with gravity return; VCL442-HV Voltage Clamp } \\
\text { pulse-width modulated resistor-loading; Windy boy } \\
6000 \mathrm{US}\end{array}$ \\
\hline
\end{tabular}

NREL measured the distance of the blade tip to the center of the rotor as $3.7 \mathrm{~m}$, which would make the diameter $7.4 \mathrm{~m}$. If the turbine has this measured rotor diameter of $7.4 \mathrm{~m}$ instead of the specified diameter of $7.2 \mathrm{~m}$, this would reduce the peak $\mathrm{Cp}$ from 0.42 to 0.40 .

The power transducer was connected between the NREL grid and the subpanel (Figure 2) to which the voltage clamp and the inverters are connected. It thus captures the combined consumption/production of all three components. 


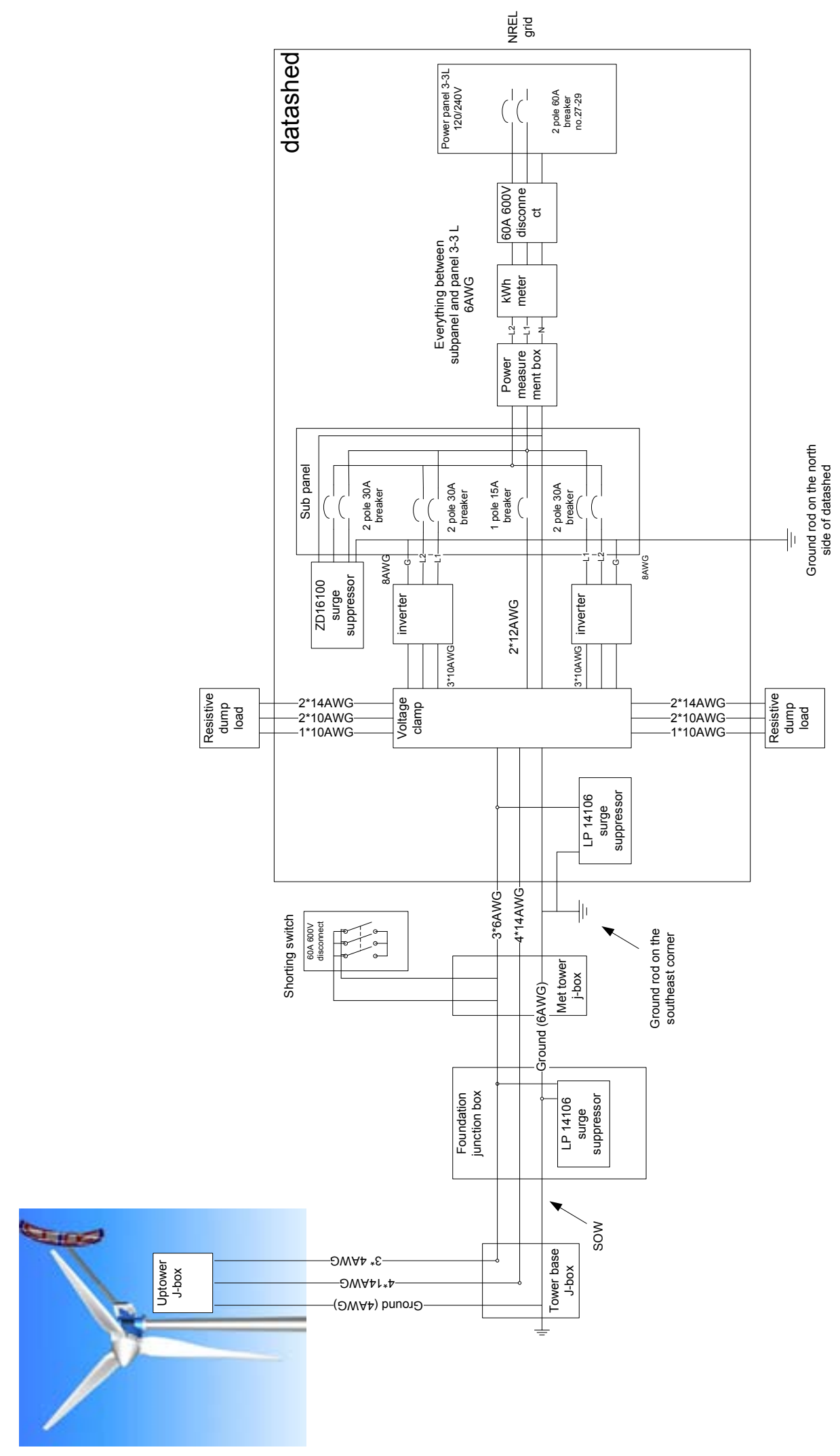

Figure 2. Electrical diagram of the ARE 442 installation 


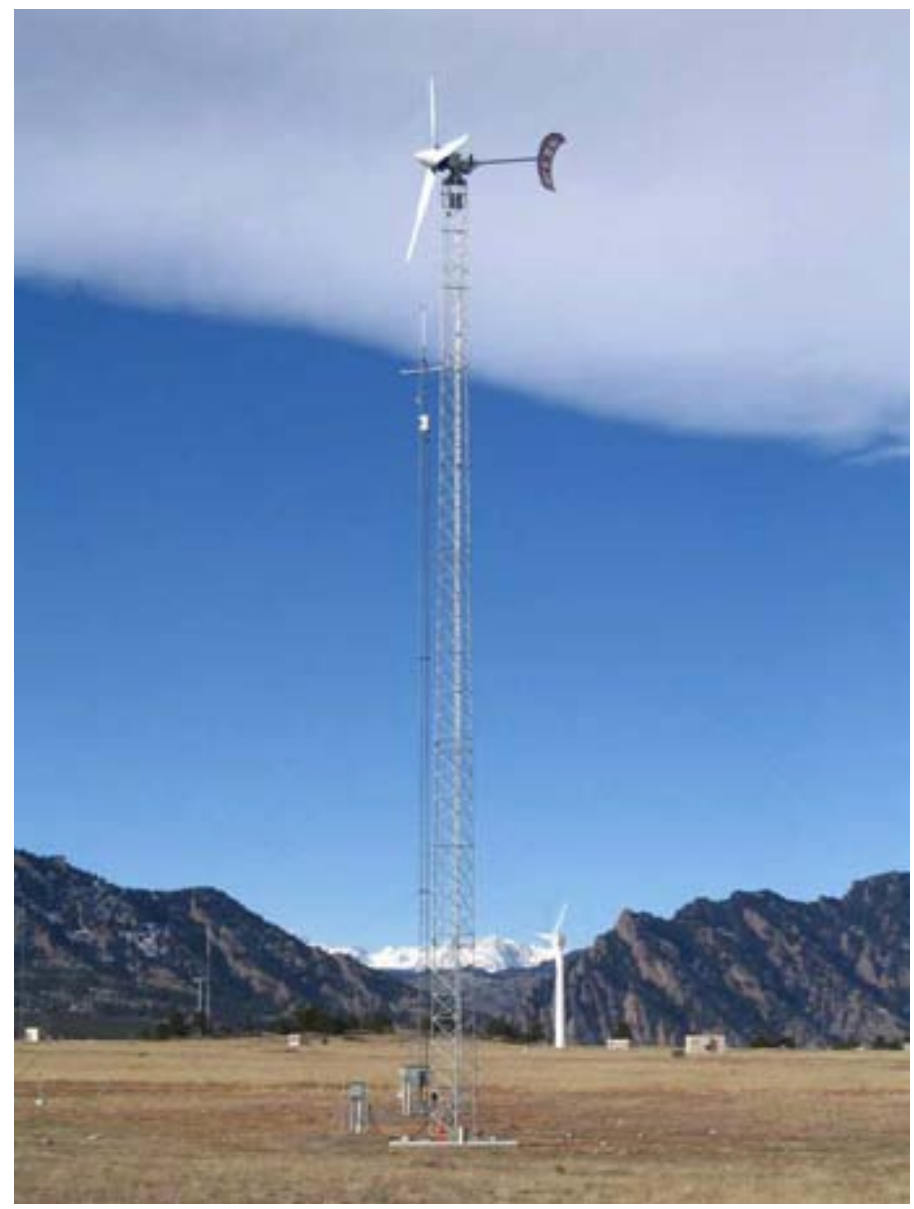

Figure 3. ARE 442 test turbine at the NWTC 


\section{Test Site Description}

The test turbine is located at site 3.3a at the NWTC located 8 miles south of Boulder, Colorado. The terrain primarily consists of mostly flat terrain with short vegetation. The test site has prevailing wind bearing $292^{\circ}$ relative to true north. For measurements where it is important to accurately measure wind speed, NREL used data obtained when wind direction is between $214^{\circ}$ and $74^{\circ}$ true. In this measurement sector, the influence of terrain and obstructions on the anemometer is small. Figure 4 shows the turbine and meteorological tower locations. This figure also shows nearby obstructions and topographical features of the site. A circle indicating 20 rotor diameters is drawn on the map. Sizes and distances of nearby obstructions are provided in Table 2.

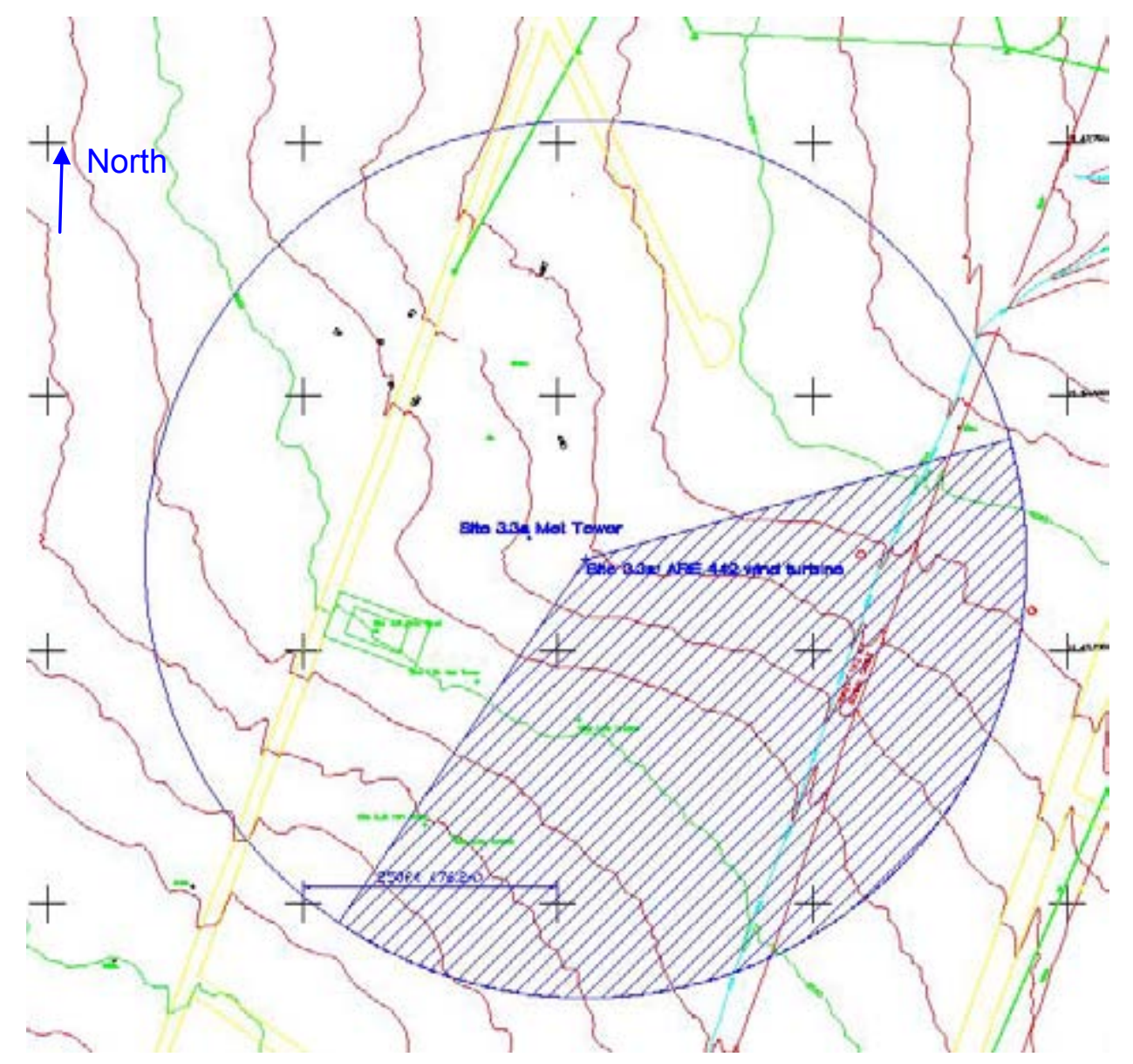

Figure 4. Map of the test site 
Table 2. Structures close to test turbine

\begin{tabular}{|c|c|c|c|c|c|c|}
\hline \multirow{2}{*}{$\begin{array}{l}\text { Obstacle or } \\
\text { Turbine }\end{array}$} & \multirow{2}{*}{$\begin{array}{l}\text { Relative } \\
\text { to: }\end{array}$} & \multirow{2}{*}{$\begin{array}{c}\text { Distance } \\
\text { (m) }\end{array}$} & \multirow{2}{*}{$\begin{array}{l}\text { Bearing } \\
(\operatorname{deg} \mathrm{T})\end{array}$} & \multirow{2}{*}{$\begin{array}{l}\text { Equiv. Dia. } \\
\text { (m) }\end{array}$} & \multicolumn{2}{|c|}{ Obstructed Sector } \\
\hline & & & & & $\begin{array}{c}\text { Start } \\
(\operatorname{deg} T)\end{array}$ & $\begin{array}{c}\text { End } \\
(\operatorname{deg} T)\end{array}$ \\
\hline Met A & Test Turbine & 18.3 & 290 & 0.8 & & \\
\hline Data Shed & Test Turbine & 62.8 & 250.6 & 4.3 & 0 & 0 \\
\hline Gaia & Test Turbine & 48.0 & 182.3 & $N / A$ & 152 & 213 \\
\hline Met B & Test Turbine & 49.2 & 221.7 & 0.8 & 0 & 0 \\
\hline Mariah & Test Turbine & 92.0 & 205.6 & $N / A$ & 0 & 0 \\
\hline Met C & Test Turbine & 93.3 & 211.2 & 0.7 & 0 & 0 \\
\hline Test Turbine & Met Tower & 18.3 & 110 & $\mathrm{~N} / \mathrm{A}$ & 73 & 147 \\
\hline Data Shed & Met Tower & 50.0 & 237 & 4.3 & 0 & 0 \\
\hline Gaia & Met Tower & 56.4 & 164 & $\mathrm{~N} / \mathrm{A}$ & 136 & 193 \\
\hline Met B & Met Tower & 45.7 & 200 & 0.8 & 0 & 0 \\
\hline Mariah & Met Tower & 92.0 & 194 & N/A & 0 & 0 \\
\hline Met C & Met Tower & 91.5 & 200 & 0.7 & 0 & 0 \\
\hline
\end{tabular}

Based upon this analysis, NREL has established a measurement sector from $214^{\circ}$ to $74^{\circ}$.

NREL completed a site assessment to determine if the site fails the requirements of Annex $A$ of the Standard and would therefore require a site calibration. Table 3 shows the results from the site assessment. Based on the site assessment results, a site calibration is not required.

Table 3. Criteria for test site without site calibration

\begin{tabular}{|l|c|c|c|c|}
\hline \multicolumn{1}{|c|}{ Description } & Distance & $\begin{array}{c}\text { Sector } \\
(\mathrm{deg})\end{array}$ & $\begin{array}{c}\text { Test Site } \\
\text { Condition }\end{array}$ & Pass/Fail \\
\hline Maximum slope of best fit plane < 3\% & $<2 \mathrm{~L}$ & 360 & $2.3 \%$ & Pass \\
\hline Maximum variation from best fit plane < 0.08 D & $<2 \mathrm{~L}$ & 360 & 0.02 & Pass \\
\hline Maximum slope of best fit plane $<5 \%$ & $2-4 \mathrm{~L}$ & $\mathrm{In}$ & $2.7 \%$ & Pass \\
\hline Maximum variation from best fit plane $<0.15 \mathrm{D}$ & $2-4 \mathrm{~L}$ & $\mathrm{In}$ & 0.02 & Pass \\
\hline Steepest slope maximum <10\% & $2-4 \mathrm{~L}$ & Out & $3.1 \%$ & Pass \\
\hline Maximum slope of best fit plane $<10 \%$ & $4-8 \mathrm{~L}$ & $\mathrm{In}$ & $2.7 \%$ & Pass \\
\hline Maximum variation from best fit plane $<0.15 \mathrm{D}$ & $4-8 \mathrm{~L}$ & $\mathrm{In}$ & 0.02 & Pass \\
\hline No neighboring and operating turbines & $<2 \mathrm{D}_{\mathrm{n}}$ & 360 & 0 & Pass \\
\hline No obstacles & $<2 \mathrm{D}_{\mathrm{e}}$ & 360 & 0 & Pass \\
\hline
\end{tabular}

$\mathrm{D}=$ test turbine rotor diameter

$\mathrm{L}=$ distance between test turbine and meteorological tower

$D_{e}=$ equivalent diameter of obstacle

In = inside preliminary measurement sector

Out $=$ outside preliminary measurement sector

The ARE442 was connected to the electrical grid at a nominal voltage of $240 \mathrm{VAC}$ at a frequency of $60 \mathrm{~Hz}$. The grid tolerances are $5 \%$ for voltage amplitude and $1 \%$ for frequency. 


\section{Description of Test Equipment}

All test equipment was calibrated; calibration sheets are included in Appendix B. Table 4 shows the equipment used and calibration due dates. Figure 5 shows placement of the meteorological instruments on the tower. The anemometer was sent out for recalibration after the test period. The difference between the two calibrations was within the tolerances allowed by the Standard. The data acquisition modules were out of calibration during the test period. They were sent out for post-test calibration and found to be within specification. The post-test calibration sheets are included in Appendix B as well.

Table 4. Equipment used in the power performance test

\begin{tabular}{|l|l|l|l|}
\hline Instrument & Make, Model & Serial Number & Calibration Due Date \\
\hline Power transducer & Secondwind Phaser 5FM-4A20 & 02663 & 28 Apr 2009 \\
\hline Current transducers & OSI 12974 & $\begin{array}{l}001235408 \\
001235411\end{array}$ & $\begin{array}{l}\text { Calibrated with power } \\
\text { transducer }\end{array}$ \\
\hline Primary anemometer & Thies, First Class & 0707886 & 28 Feb 2009 \\
\hline Reference anemometer & NRG, Max 40 & 179500049022 & In situ \\
\hline Wind vane & Met One, 020C with aluminum & G4706 & 28 Feb 2009 \\
& vane & & \\
\hline Pressure sensor & Vaisala, PTB101B & T4730007 & 26 Aug 2009 \\
\hline Temperature sensor & Met One, T-200 & 0789020 & 10 Oct 2009 \\
\hline Precipitation sensor & Campbell Scientific, 237 & None & In situ \\
\hline $\begin{array}{l}\text { Data acquisition } \\
\text { system }\end{array}$ & $\begin{array}{l}\text { Compact DAQ w/LabView-based } \\
\text { data acquisition }\end{array}$ & & \\
cDAQ-9172 & 12EAE14 & 31 May 2008 \\
& NI 9229 & $\begin{array}{l}\text { NA2037 } \\
12 C 73 B 4\end{array}$ & 3 Aug 2008 \\
NI 9217 & Oct 2008 \\
\hline
\end{tabular}




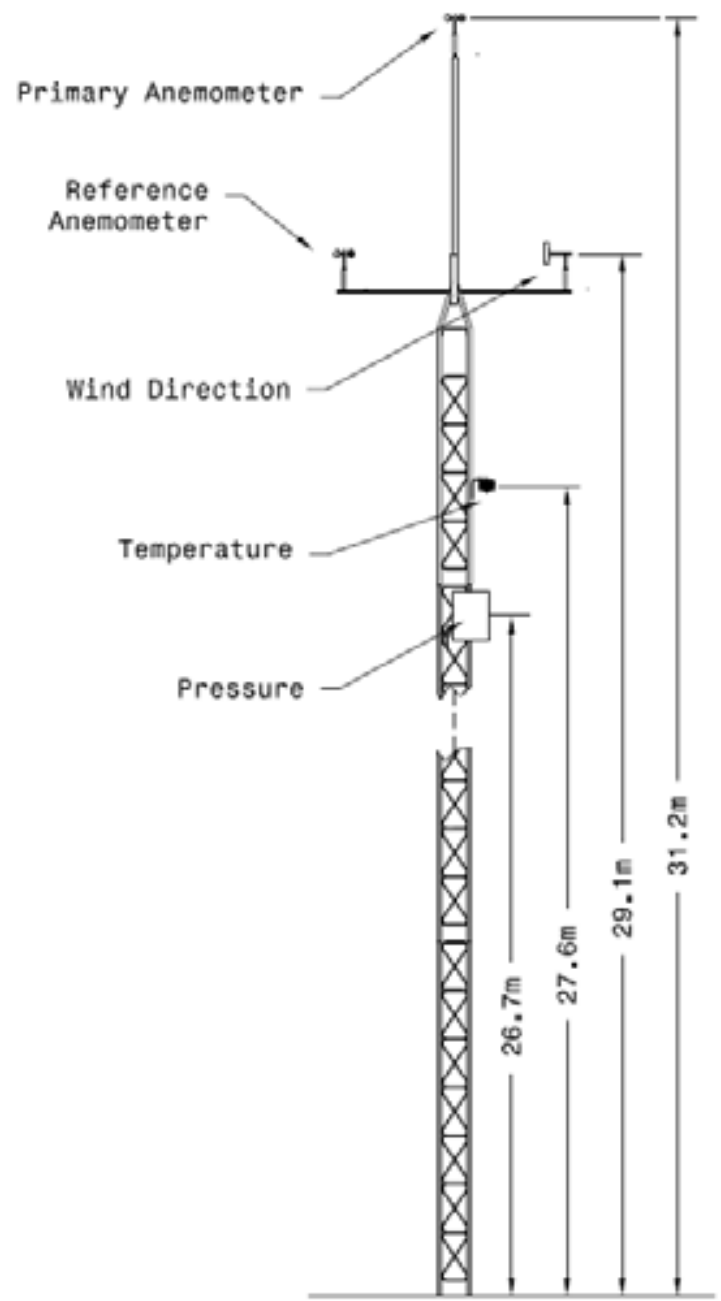

Figure 5. Meteorological tower and instruments

Figure 5 shows placement of the meteorological instruments on the tower. (Note that the Primary Anemometer is within the allowable $2.5 \%$ of hub height. To ensure that only data obtained during normal operation of the turbine are used in the analysis, and to ensure data are not corrupted, data sets are excluded from the database under the following circumstances:

- external conditions other than wind speed are out of the normal range for turbine operation,

- turbine cannot operate because of a turbine fault condition, and,

- turbine is manually shut down or in a test or maintenance operating mode.

Two methods were used to track when any of these conditions occur during the test. With the first method, the logbook was checked for such events. For the second, the turbine controller had a status signal which was measured that indicates when the turbine is available or braked. A copy of the logbook is available upon request. No maintenance was performed during the test period. 


\section{Description of Test Procedure}

The test was conducted according to the procedures in the Standard. The sampling rate was $10 \mathrm{kHz}$, which was then decimated to $1 \mathrm{~Hz}$ data. The averaging time was 1 minute for the mean values. Standard deviation, minimum, and maximum values for each averaging period were also collected.

The turbine status signal for the ARE 442 was obtained by checking the release of the up-tower brake relay. The status signal indicated if the turbine was braked or not.

Only database A is reported since the ARE 442 does not have a cut-out wind speed.

Table 5. Uncertainty values used in the analysis

\begin{tabular}{|c|c|c|}
\hline Component & Uncertainty & Source \\
\hline \multicolumn{3}{|l|}{ Power (Inverter) } \\
\hline voltage transducer & NA & \\
\hline current sensor/signal & $12 \mathrm{~W}$ & Specifications (specs) \\
\hline power transducer * & $0.12 \%$ & Specs \\
\hline data acquisition & $17 W+0.08 \%$ & Specs \\
\hline resistor & $0.01 \%$ & Specs \\
\hline \multicolumn{3}{|l|}{ Wind Speed } \\
\hline calibration & $0.02 \mathrm{~m} / \mathrm{s}$ & Calibration sheet \\
\hline operational characteristics & $0.05 \mathrm{~m} / \mathrm{s}+0.52 \%$ & IEC \\
\hline mounting effects & $1.00 \%$ & Assumption \\
\hline terrain effects & $2.00 \%$ & IEC \\
\hline data acquisition & $<0.01 \mathrm{~m} / \mathrm{s}$ & Assumption \\
\hline \multicolumn{3}{|l|}{ Temperature } \\
\hline temperature sensor & $0.15^{\circ} \mathrm{C}$ & Specs \\
\hline radiation shielding & $1.15^{\circ} \mathrm{C}$ & Assumption \\
\hline mounting effects & $0.11^{\circ} \mathrm{C}$ & IEC method \\
\hline algorithm & $0.00^{\circ} \mathrm{C}$ & Included in DAS \\
\hline data acquisition & $0.35^{\circ} \mathrm{C}$ & Specs \\
\hline \multicolumn{3}{|l|}{ Air Pressure } \\
\hline pressure sensor & $0.20 \mathrm{kPa}$ & Instrument specs. \\
\hline mounting effects & $<0.01 \mathrm{kPa}$ & IEC method \\
\hline data acquisition & $0.06 \mathrm{kPa}$ & Specs \\
\hline
\end{tabular}




\section{Test Results}

\subsection{Tabular Results of Power Performance Test}

Table 6 through 9 provide the power performance test results in tabular format. 
Table 6. Performance at sea-level air density; $1.225 \mathrm{~kg} / \mathrm{m}^{3}$

\begin{tabular}{|c|c|c|c|c|c|c|c|}
\hline \multicolumn{8}{|c|}{ Measured power curve (database A) } \\
\hline \multicolumn{4}{|c|}{ Reference air density: $1.225 \mathrm{~kg} / \mathrm{m}^{3}$} & \multirow[b]{2}{*}{$\begin{array}{c}\text { Number of } \\
\text { 1-Minute } \\
\text { Data Sets }\end{array}$} & \multirow{2}{*}{$\begin{array}{c}\text { Category A } \\
\text { Standard } \\
\text { Uncertainty } \\
\text { (kW) }\end{array}$} & \multirow{2}{*}{\begin{tabular}{|c|} 
Category B \\
Standard \\
Uncertainty \\
(kW)
\end{tabular}} & \multirow{2}{*}{$\begin{array}{c}\text { Combined } \\
\text { Standard } \\
\text { Uncertainty } \\
\text { (kW) }\end{array}$} \\
\hline $\begin{array}{l}\text { Bin } \\
(\mathrm{m} / \mathrm{s})\end{array}$ & $\begin{array}{c}\text { Normalized } \\
\text { Wind Speed } \\
(\mathrm{m} / \mathrm{s})\end{array}$ & $\begin{array}{l}\text { Power } \\
\text { Output } \\
\text { (kW) }\end{array}$ & $\mathrm{C}_{\mathrm{p}}$ & & & & \\
\hline 0.5 & 0.51 & -0.03 & -9.00 & 1000 & 0.00 & 0.16 & 0.16 \\
\hline 1 & 1.00 & -0.04 & -1.61 & 1210 & 0.00 & 0.16 & 0.16 \\
\hline 1.5 & 1.50 & -0.04 & -0.53 & 1339 & 0.00 & 0.16 & 0.16 \\
\hline 2. & 1.99 & -0.05 & -0.27 & 1423 & 0.00 & 0.16 & 0.16 \\
\hline 2.5 & 2.50 & -0.05 & -0.13 & 1196 & 0.00 & 0.16 & 0.16 \\
\hline 3 & 2.98 & -0.01 & -0.02 & 1061 & 0.00 & 0.16 & 0.16 \\
\hline 3.5 & 3.49 & 0.08 & 0.08 & 861 & 0.01 & 0.16 & 0.16 \\
\hline 4 & 4.00 & 0.30 & 0.19 & 713 & 0.01 & 0.17 & 0.17 \\
\hline 4.5 & 4.49 & 0.59 & 0.26 & 647 & 0.01 & 0.18 & 0.18 \\
\hline 5 & 5.00 & 0.91 & 0.29 & 579 & 0.02 & 0.18 & 0.18 \\
\hline 5.5 & 5.51 & 1.35 & 0.32 & 604 & 0.02 & 0.20 & 0.20 \\
\hline 6 & 6.00 & 1.86 & 0.35 & 586 & 0.02 & 0.23 & 0.23 \\
\hline 6.5 & 6.50 & 2.42 & 0.35 & 624 & 0.02 & 0.24 & 0.24 \\
\hline 7 & 7.00 & 3.04 & 0.35 & 647 & 0.02 & 0.27 & 0.27 \\
\hline 7.5 & 7.49 & 3.70 & 0.35 & 599 & 0.02 & 0.29 & 0.29 \\
\hline 8 & 8.00 & 4.41 & 0.35 & 535 & 0.02 & 0.32 & 0.32 \\
\hline 8.5 & 8.49 & 5.19 & 0.34 & 544 & 0.02 & 0.36 & 0.36 \\
\hline 9 & 9.00 & 6.02 & 0.33 & 478 & 0.02 & 0.39 & 0.39 \\
\hline 9.5 & 9.50 & 6.81 & 0.32 & 416 & 0.03 & 0.39 & 0.39 \\
\hline 10 & 10.00 & 7.70 & 0.31 & 404 & 0.03 & 0.45 & 0.45 \\
\hline 10.5 & 10.49 & 8.47 & 0.29 & 384 & 0.04 & 0.42 & 0.42 \\
\hline 11 & 10.99 & 9.17 & 0.28 & 362 & 0.04 & 0.40 & 0.40 \\
\hline 11.5 & 11.50 & 9.69 & 0.26 & 340 & 0.05 & 0.32 & 0.33 \\
\hline 12 & 12.01 & 10.10 & 0.23 & 289 & 0.05 & 0.29 & 0.29 \\
\hline 12.5 & 12.49 & 10.05 & 0.21 & 245 & 0.06 & 0.17 & 0.19 \\
\hline 13 & 12.97 & 9.91 & 0.18 & 243 & 0.06 & 0.20 & 0.21 \\
\hline 13.5 & 13.50 & 9.42 & 0.15 & 186 & 0.06 & 0.33 & 0.34 \\
\hline 14 & 14.00 & 9.00 & 0.13 & 162 & 0.05 & 0.33 & 0.33 \\
\hline 14.5 & 14.48 & 8.58 & 0.11 & 138 & 0.04 & 0.34 & 0.34 \\
\hline 15 & 15.00 & 8.28 & 0.10 & 124 & 0.06 & 0.26 & 0.27 \\
\hline 15.5 & 15.54 & 7.86 & 0.08 & 74 & 0.11 & 0.33 & 0.35 \\
\hline 16 & 15.99 & 7.85 & 0.08 & 78 & 0.06 & 0.17 & 0.18 \\
\hline 16.5 & 16.49 & 7.59 & 0.07 & 47 & 0.05 & 0.26 & 0.27 \\
\hline 17 & 16.95 & 7.33 & 0.06 & 33 & 0.14 & 0.28 & 0.31 \\
\hline 17.5 & 17.51 & 7.48 & 0.06 & 27 & 0.14 & 0.20 & 0.25 \\
\hline 18 & 18.01 & 7.57 & 0.05 & 24 & 0.08 & 0.18 & 0.20 \\
\hline
\end{tabular}


Table 7. Performance at site average density; $1.05 \mathrm{~kg} / \mathrm{m}^{3}$

\begin{tabular}{|c|c|c|c|c|c|c|c|}
\hline \multicolumn{8}{|c|}{ Measured power curve (database A) } \\
\hline \multicolumn{4}{|c|}{ Reference air density: $1.05 \mathrm{~kg} / \mathrm{m}^{3}$} & \multirow[b]{2}{*}{$\begin{array}{c}\text { Number of } \\
\text { 1-Minute } \\
\text { Data Sets }\end{array}$} & \multirow{2}{*}{$\begin{array}{c}\text { Category A } \\
\text { Standard } \\
\text { Uncertainty } \\
(\mathrm{kW})\end{array}$} & \multirow{2}{*}{\begin{tabular}{|c|} 
Category B \\
Standard \\
Uncertainty \\
(kW)
\end{tabular}} & \multirow{2}{*}{$\begin{array}{c}\text { Combined } \\
\text { Standard } \\
\text { Uncertainty } \\
\text { (kW) }\end{array}$} \\
\hline $\begin{array}{l}\text { Bin } \\
(\mathrm{m} / \mathrm{s})\end{array}$ & $\begin{array}{c}\text { Normalized } \\
\text { Wind Speed } \\
(\mathrm{m} / \mathrm{s})\end{array}$ & $\begin{array}{c}\text { Power } \\
\text { Output } \\
\text { (kW) }\end{array}$ & $C_{p}$ & & & & \\
\hline 0.5 & 0.52 & -0.03 & -10.29 & 928 & 0.00 & 0.16 & 0.16 \\
\hline 1 & 1.00 & -0.04 & -1.84 & 1129 & 0.00 & 0.16 & 0.16 \\
\hline 1.5 & 1.50 & -0.05 & -0.63 & 1254 & 0.00 & 0.16 & 0.16 \\
\hline 2 & 2.00 & -0.05 & -0.29 & 1376 & 0.00 & 0.16 & 0.16 \\
\hline 2.5 & 2.50 & -0.05 & -0.16 & 1175 & 0.00 & 0.16 & 0.16 \\
\hline 3 & 2.99 & -0.03 & -0.05 & 1079 & 0.00 & 0.16 & 0.16 \\
\hline 3.5 & 3.49 & 0.04 & 0.05 & 862 & 0.00 & 0.16 & 0.16 \\
\hline 4 & 3.99 & 0.19 & 0.14 & 714 & 0.01 & 0.17 & 0.17 \\
\hline 4.5 & 4.50 & 0.46 & 0.24 & 646 & 0.01 & 0.17 & 0.17 \\
\hline 5 & 5.00 & 0.73 & 0.27 & 574 & 0.01 & 0.18 & 0.18 \\
\hline 5.5 & 5.50 & 1.11 & 0.31 & 549 & 0.02 & 0.19 & 0.19 \\
\hline 6 & 6.00 & 1.53 & 0.33 & 585 & 0.02 & 0.21 & 0.21 \\
\hline 6.5 & 6.50 & 2.04 & 0.35 & 546 & 0.02 & 0.23 & 0.23 \\
\hline 7 & 7.00 & 2.59 & 0.35 & 605 & 0.02 & 0.25 & 0.25 \\
\hline 7.5 & 7.49 & 3.19 & 0.35 & 618 & 0.02 & 0.27 & 0.27 \\
\hline 8 & 7.99 & 3.84 & 0.35 & 550 & 0.02 & 0.30 & 0.30 \\
\hline 8.5 & 8.50 & 4.51 & 0.34 & 503 & 0.02 & 0.32 & 0.32 \\
\hline 9 & 8.98 & 5.25 & 0.34 & 514 & 0.02 & 0.36 & 0.37 \\
\hline 9.5 & 9.50 & 6.06 & 0.33 & 459 & 0.02 & 0.39 & 0.39 \\
\hline 10 & 10.00 & 6.82 & 0.32 & 398 & 0.03 & 0.39 & 0.39 \\
\hline 10.5 & 10.51 & 7.67 & 0.31 & 388 & 0.03 & 0.45 & 0.45 \\
\hline 11 & 11.00 & 8.41 & 0.30 & 361 & 0.04 & 0.42 & 0.42 \\
\hline 11.5 & 11.49 & 9.04 & 0.28 & 344 & 0.04 & 0.39 & 0.39 \\
\hline 12 & 12.00 & 9.62 & 0.26 & 327 & 0.05 & 0.36 & 0.37 \\
\hline 12.5 & 12.50 & 10.02 & 0.24 & 283 & 0.06 & 0.29 & 0.30 \\
\hline 13 & 12.98 & 10.06 & 0.22 & 250 & 0.06 & 0.17 & 0.19 \\
\hline 13.5 & 13.49 & 10.03 & 0.19 & 243 & 0.06 & 0.17 & 0.19 \\
\hline 14 & 13.99 & 9.61 & 0.16 & 186 & 0.06 & 0.32 & 0.33 \\
\hline 14.5 & 14.50 & 9.16 & 0.14 & 166 & 0.05 & 0.34 & 0.34 \\
\hline 15 & 14.99 & 8.83 & 0.12 & 143 & 0.05 & 0.29 & 0.29 \\
\hline 15.5 & 15.48 & 8.44 & 0.11 & 126 & 0.05 & 0.33 & 0.33 \\
\hline 16 & 15.98 & 8.14 & 0.09 & 89 & 0.07 & 0.28 & 0.29 \\
\hline 16.5 & 16.52 & 7.80 & 0.08 & 80 & 0.10 & 0.30 & 0.32 \\
\hline 17 & 16.98 & 7.77 & 0.07 & 64 & 0.07 & 0.17 & 0.18 \\
\hline 17.5 & 17.48 & 7.56 & 0.07 & 43 & 0.05 & 0.24 & 0.24 \\
\hline 18 & 17.94 & 7.24 & 0.06 & 27 & 0.20 & 0.33 & 0.39 \\
\hline 18.5 & 18.48 & 7.60 & 0.06 & 26 & 0.10 & 0.33 & 0.34 \\
\hline 19 & 18.98 & 7.53 & 0.05 & 22 & 0.08 & 0.18 & 0.20 \\
\hline
\end{tabular}


Table 8. Annual energy production (AEP) at sea-level density; $1.225 \mathrm{~kg} / \mathrm{m}^{3}$

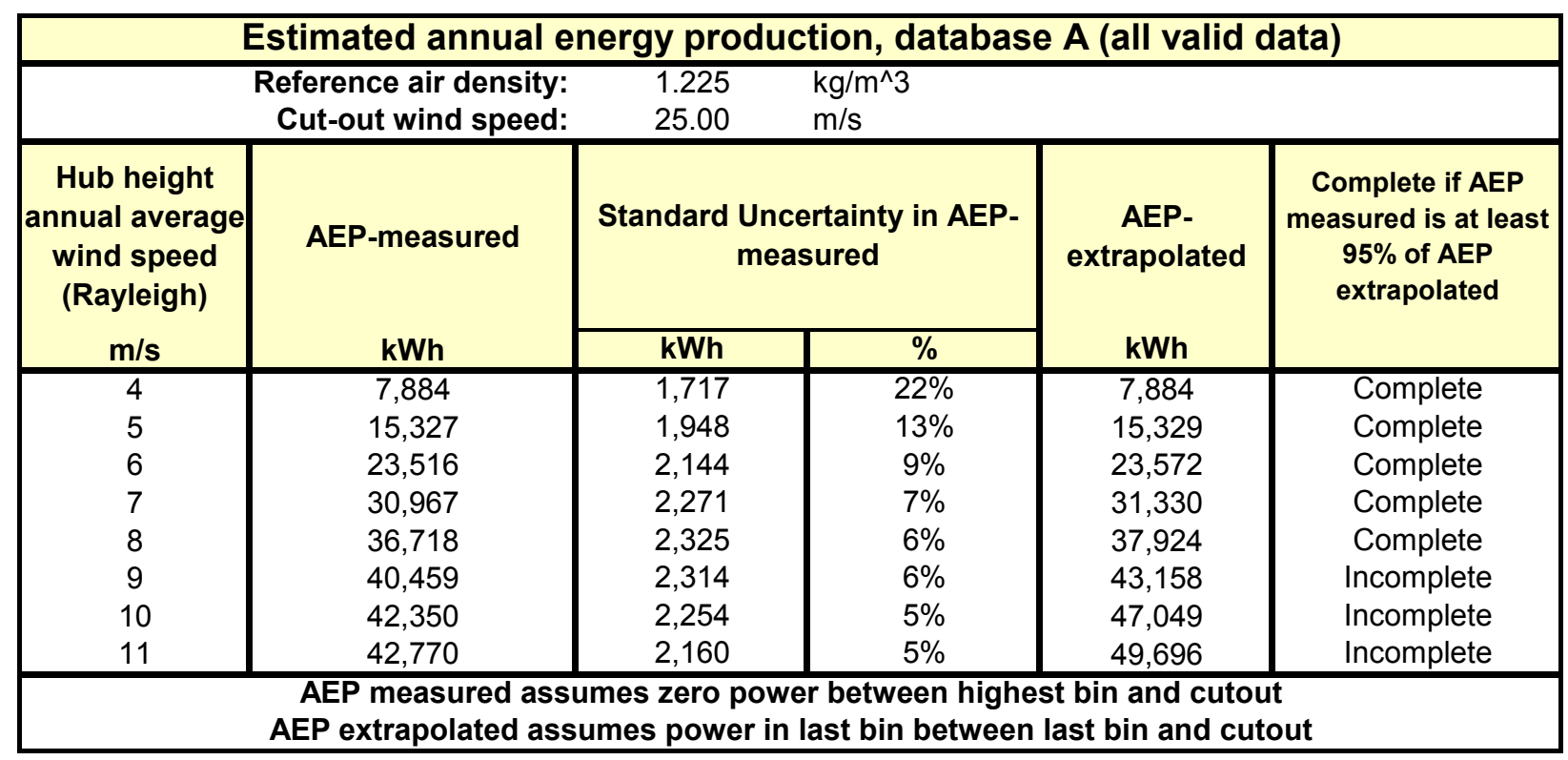

Table 9. Annual energy production at site average density; $1.05 \mathrm{~kg} / \mathrm{m}^{3}$

\begin{tabular}{|c|c|c|c|c|c|}
\hline \multicolumn{6}{|c|}{ Estimated annual energy production, database A (all valid data) } \\
\hline & $\begin{array}{l}\text { Reference air density: } \\
\text { Cut-out wind speed: }\end{array}$ & $\begin{array}{c}1.050 \\
25.0\end{array}$ & $\begin{array}{l}\mathrm{kg} / \mathrm{m}^{\wedge} 3 \\
\mathrm{~m} / \mathrm{s}\end{array}$ & & \\
\hline \multirow{2}{*}{$\begin{array}{c}\text { Hub height } \\
\text { annual average } \\
\text { wind speed } \\
\text { (Rayleigh) } \\
\text { m/s }\end{array}$} & \multirow[t]{2}{*}{ AEP-measured } & \multicolumn{2}{|c|}{$\begin{array}{c}\text { Standard Uncertainty in AEP- } \\
\text { measured }\end{array}$} & \multirow{2}{*}{$\begin{array}{c}\text { AEP- } \\
\text { extrapolated } \\
\text { kWh }\end{array}$} & \multirow{2}{*}{$\begin{array}{c}\text { Complete if AEP } \\
\text { measured is at least } \\
95 \% \text { of AEP } \\
\text { extrapolated }\end{array}$} \\
\hline & & kWh & $\%$ & & \\
\hline 4 & 6,608 & 1,670 & $25 \%$ & 6,608 & Complete \\
\hline 5 & 13,321 & 1,887 & $14 \%$ & 13,322 & Complete \\
\hline 6 & 21,066 & 2,086 & $10 \%$ & 21,091 & Complete \\
\hline 7 & 28,513 & 2,230 & $8 \%$ & 28,714 & Complete \\
\hline 8 & 34,658 & 2,310 & $7 \%$ & 35,419 & Complete \\
\hline 9 & 39,036 & 2,329 & $6 \%$ & 40,886 & Complete \\
\hline 10 & 41,641 & 2,297 & $6 \%$ & 45,046 & Incomplete \\
\hline 11 & 42,734 & 2,228 & $5 \%$ & 47,953 & Incomplete \\
\hline & AEP measure & les $2 \mathrm{er}$ & . & . & \\
\hline
\end{tabular}




\subsection{Graphical Results}

Figure 6 through 9 show the results of the power performance test in graphical format. The $12-\mathrm{kW}$ ceiling of the power maxima in Figure 8 is caused by the maximum output capability of the inverters. Figure 10 through 12 show plots of turbulence intensity and rotor speed.

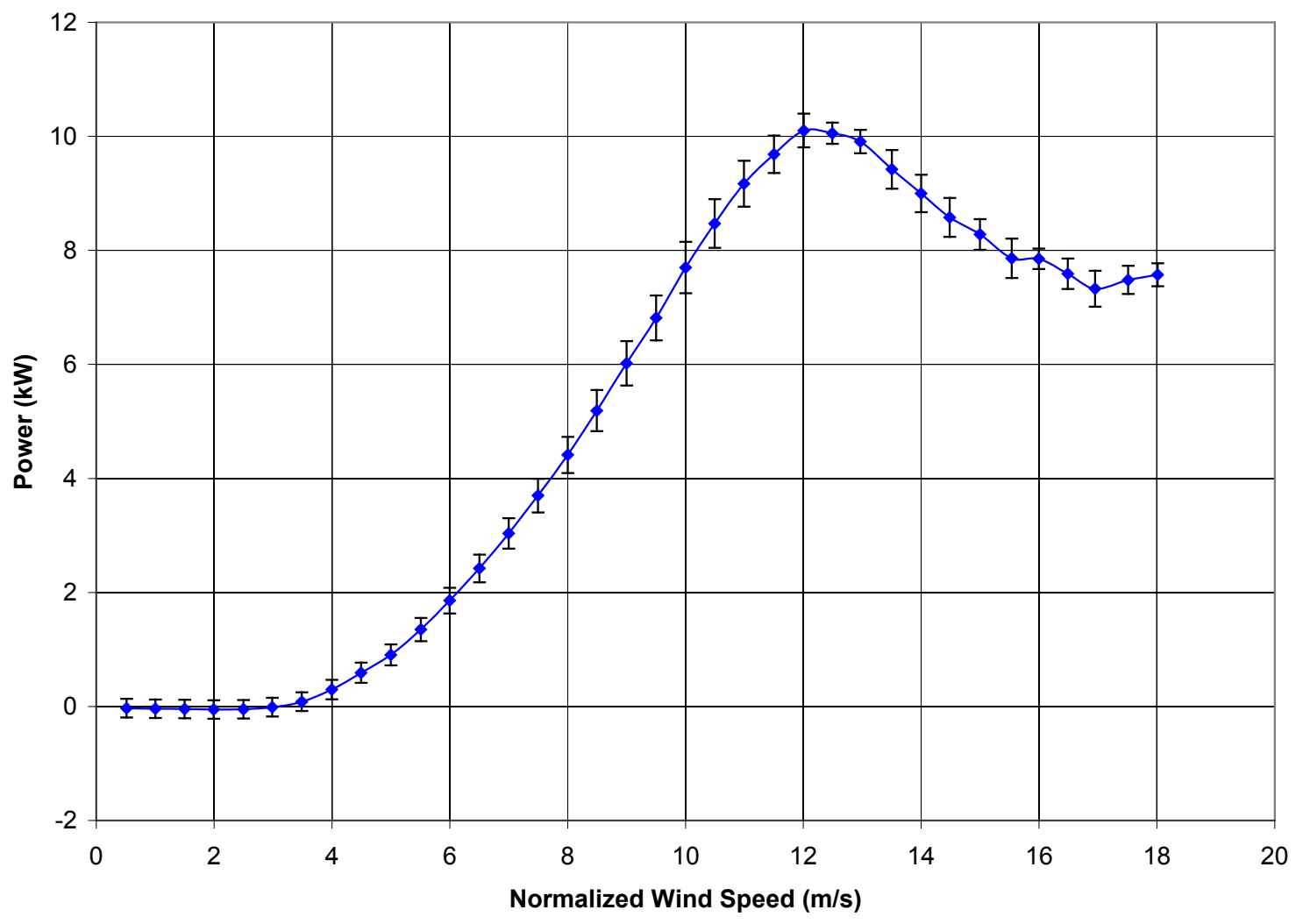

Figure 6. Power curve at sea-level density; $1.225 \mathrm{~kg} / \mathrm{m}^{3}$ 


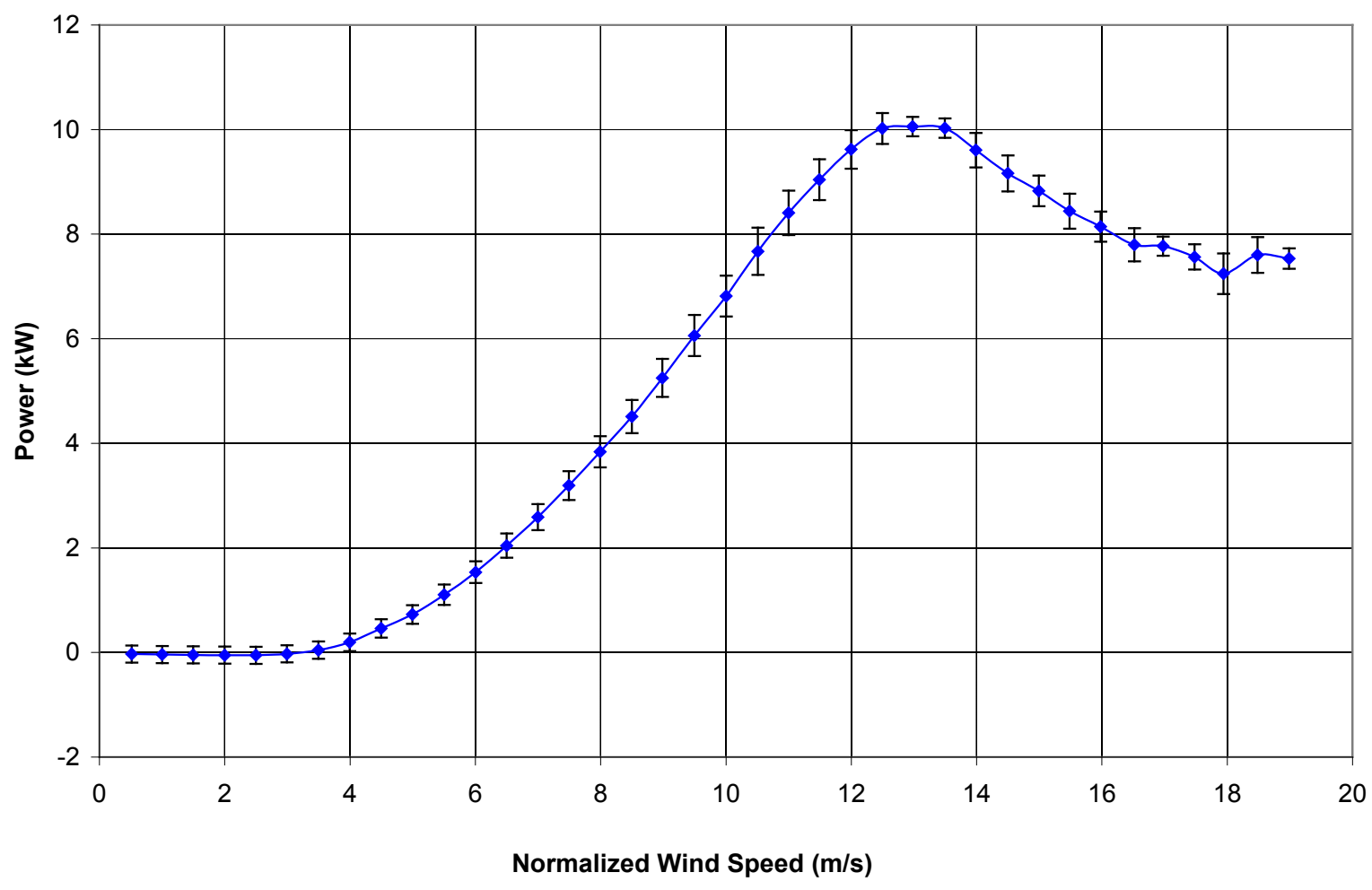

Figure 7. Power curve at site average density; $1.05 \mathrm{~kg} / \mathrm{m}^{3}$ 


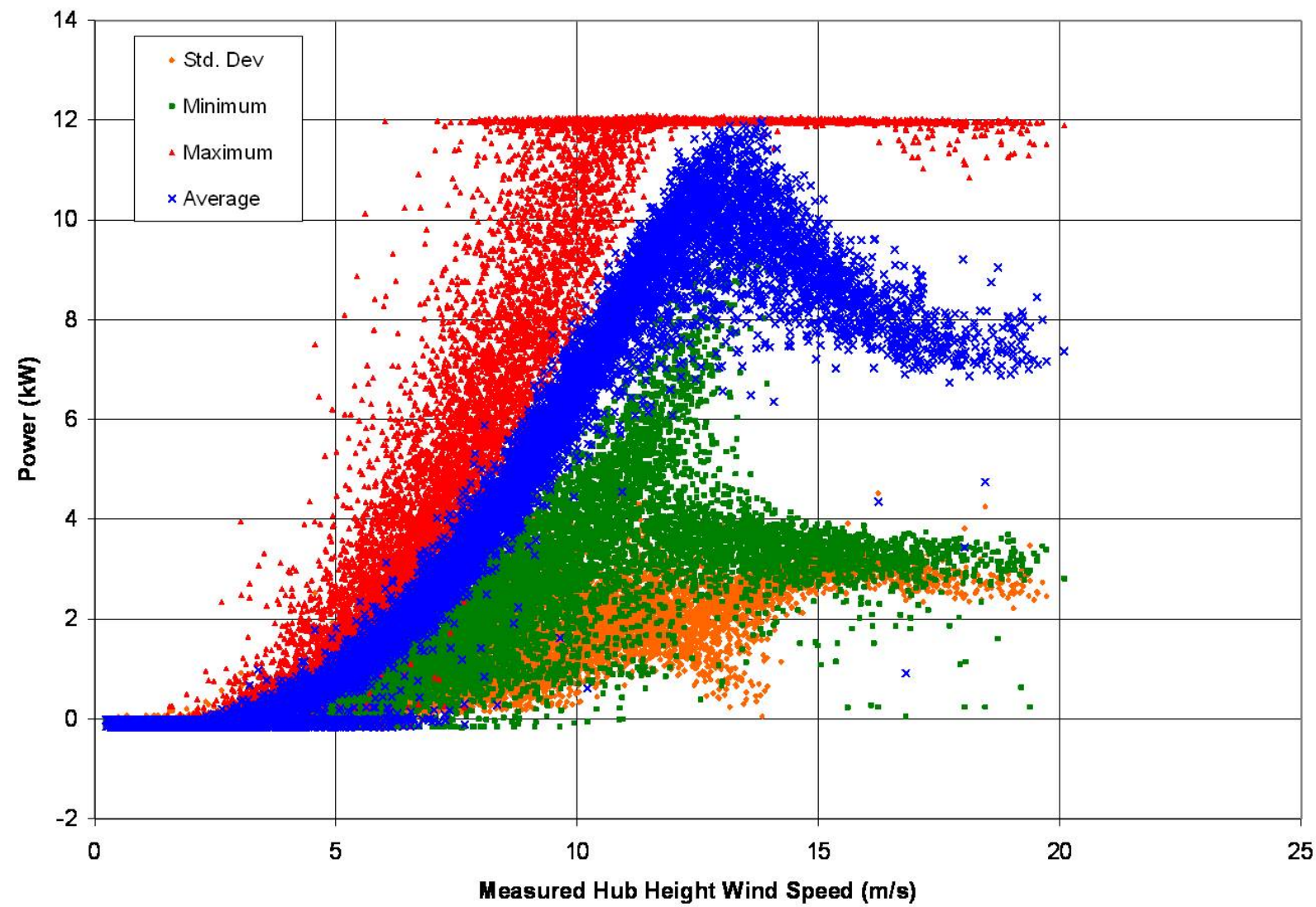

Figure 8. Scatter plot of mean, standard deviation, minimum, and maximum power data. $1 \mathrm{~Hz}$ samples with 1 minute averaging. 


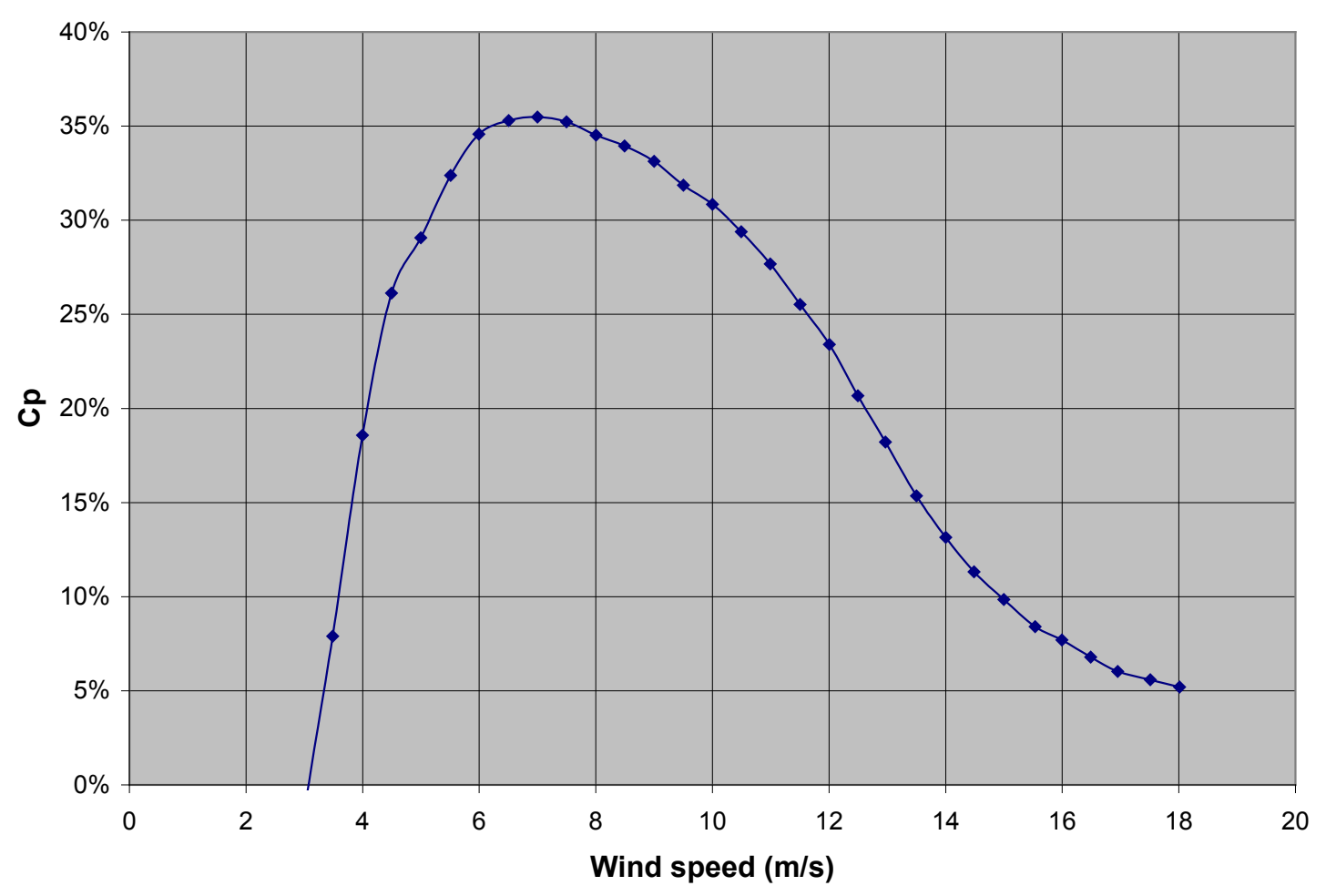

Figure 9. Coefficient of performance at sea level density; $1.225 \mathrm{~kg} / \mathrm{m}^{3}$ 


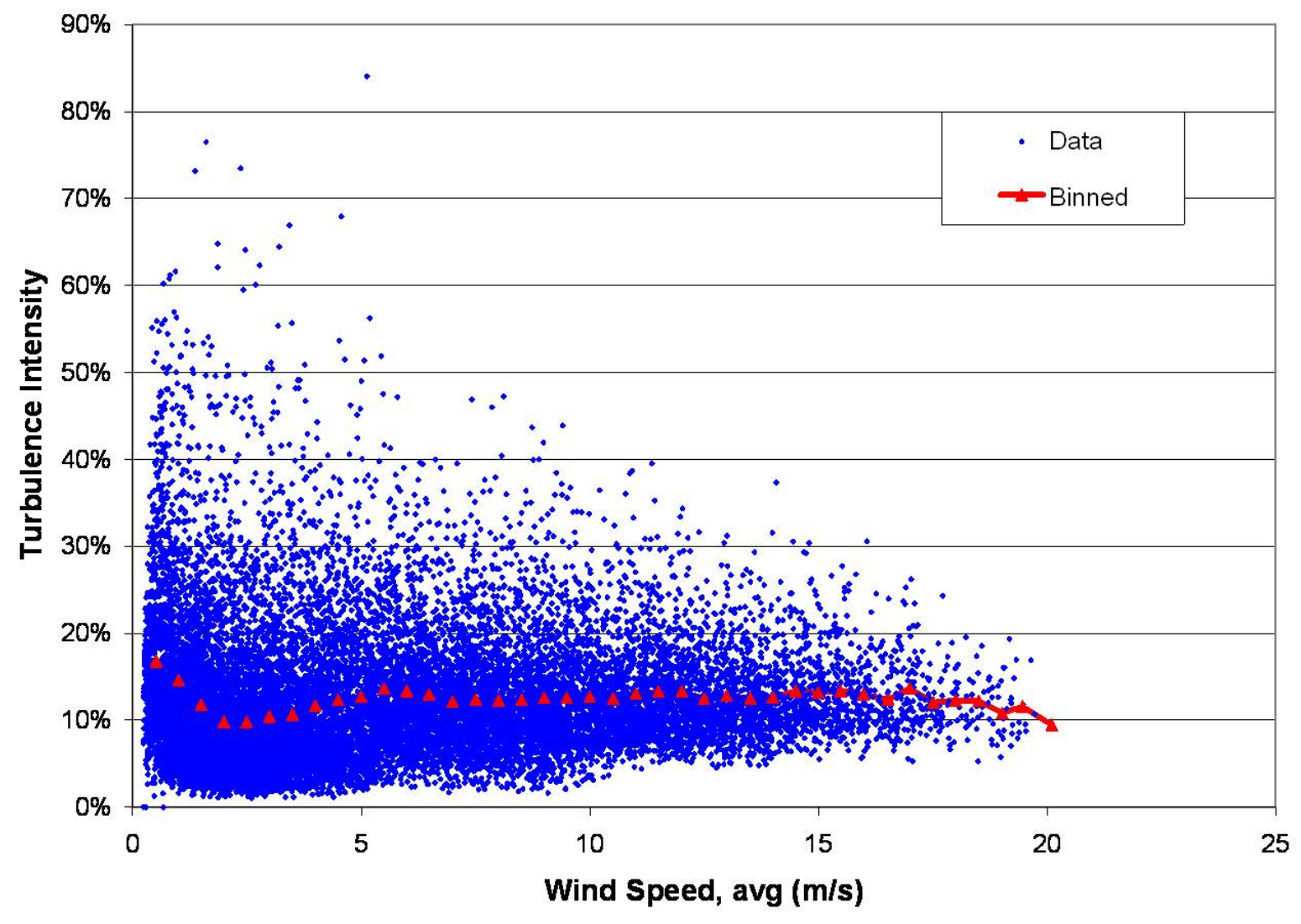

Figure 10. Wind turbulence intensity as a function of wind speed 


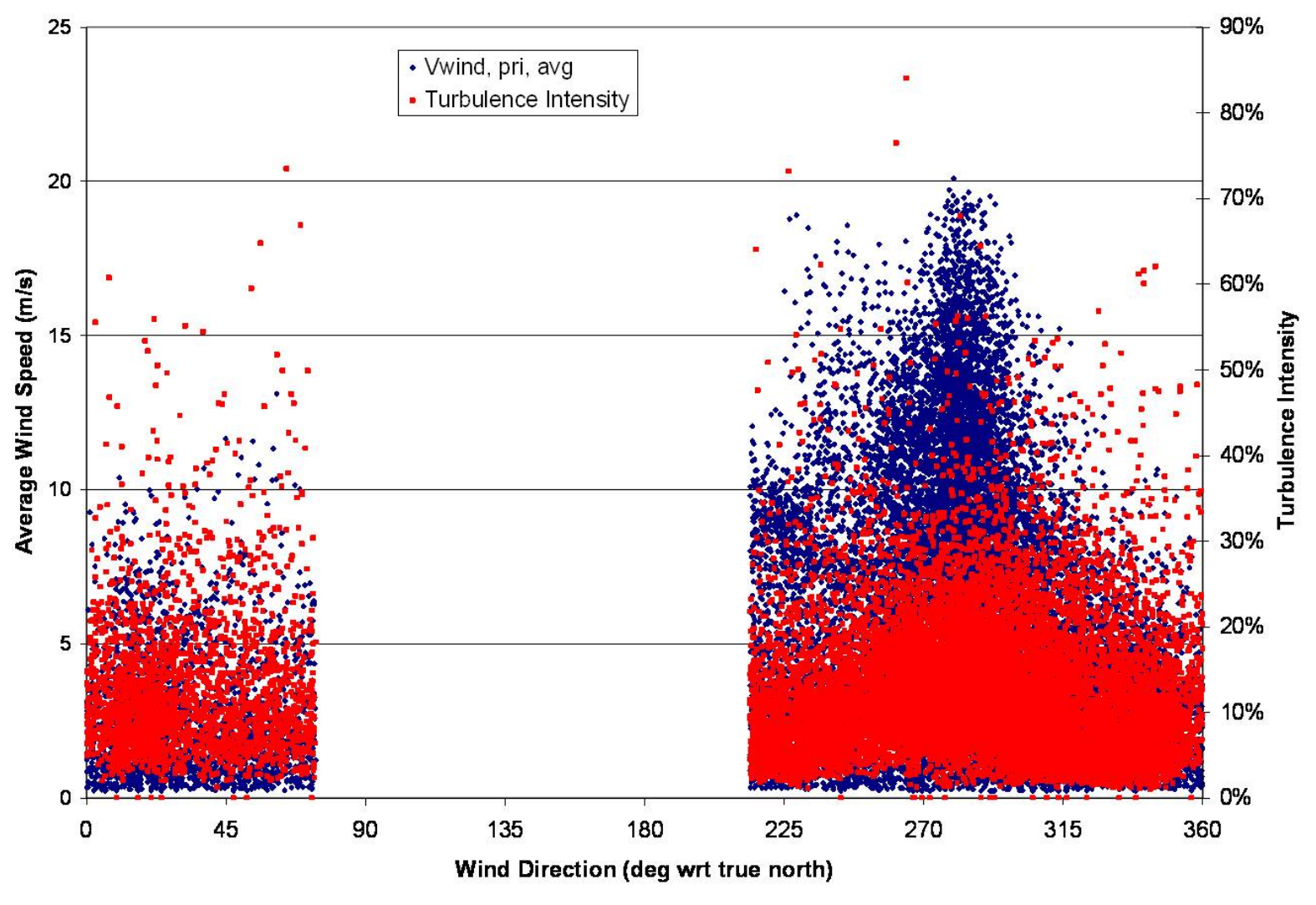

Figure 11. Wind speed and turbulence intensity as a function of wind direction 
\begin{tabular}{|l|l|r|r|r|r|r|r|r|r|r|r|r|r|r|r|r|r|r|r|r|r|}
\hline Wind speed & {$[\mathrm{m} / \mathrm{s}]$} & 0.5 & 1.0 & 1.5 & 2.0 & 2.5 & 3.0 & 3.5 & 4.0 & 4.5 & 5.0 & 5.5 & 6.0 & 6.5 & 7.0 & 7.5 & 8.0 & 8.5 & 9.0 & 9.5 & 10.0 \\
\hline
\end{tabular}

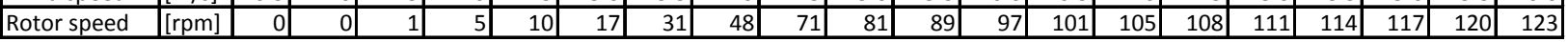

\begin{tabular}{|l|l|l|r|r|r|r|r|r|r|r|r|r|r|r|r|r|r|r|r|r|r|}
\hline Wind speed & {$[\mathrm{m} / \mathrm{s}]$} & 10.5 & 11.0 & 11.5 & 12.0 & 12.5 & 13.0 & 13.5 & 14.0 & 14.5 & 15.0 & 15.5 & 16.0 & 16.5 & 17.0 & 17.5 & 18.0 & 18.5 & 19.0 & 19.5 & 20.1 \\
\hline
\end{tabular}

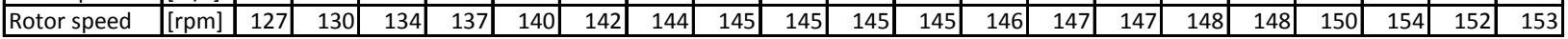

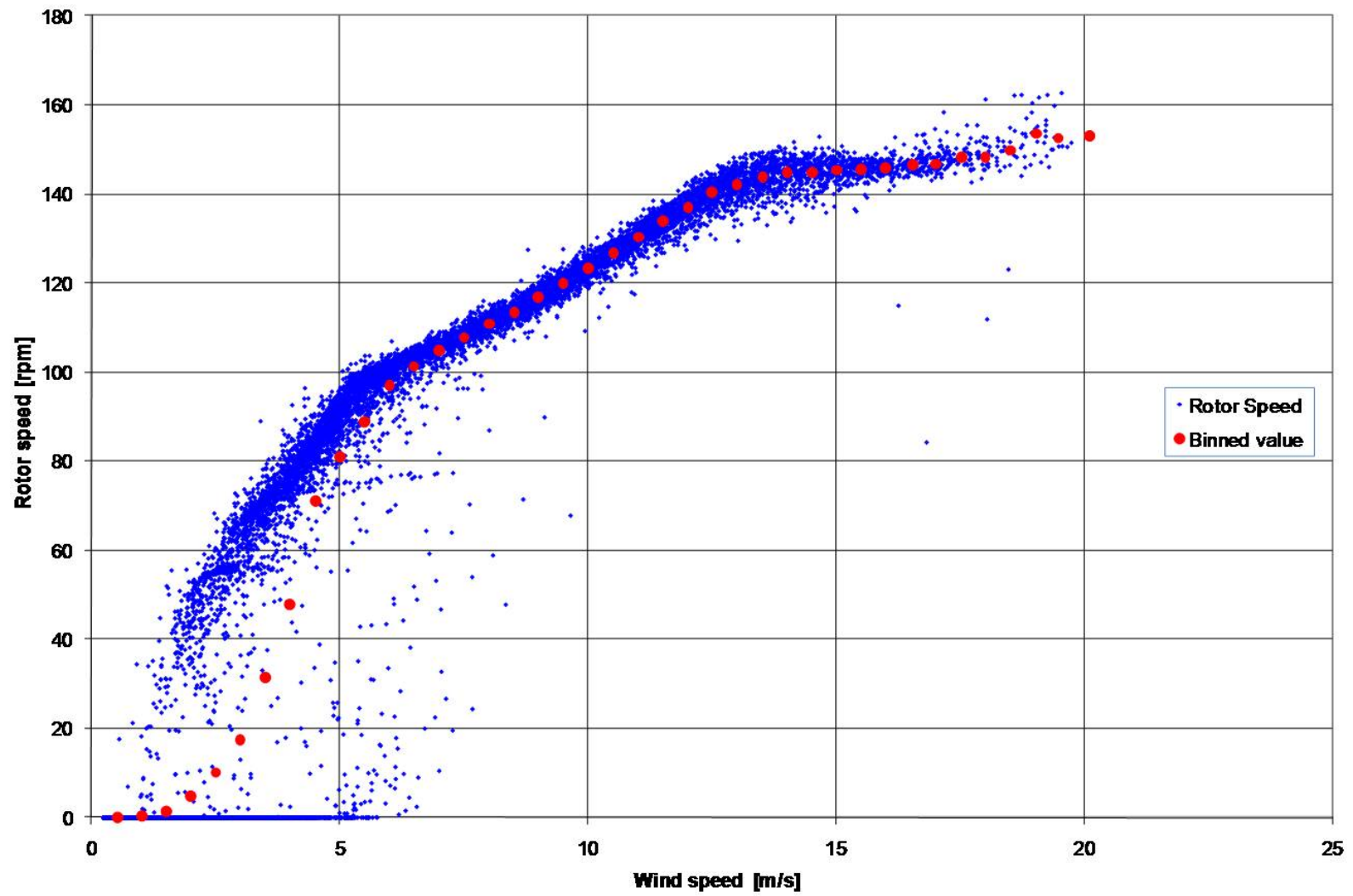

Figure 12. Rotor speed as a function of wind speed (1-minute averages) and binned values 


\section{Deviations and Exceptions}

\subsection{Deviations from the Standard}

The current transformers are not listed as compliant to IEC 60044-1, but do exceed the minimum accuracy required by the Standard.

\subsection{Exceptions to NWTC-CT Quality Assurance System}

During the test period, the data acquisition system was out of calibration. A post test calibration has been performed which showed that the system was within specification without adjustment. Thus, it can be assumed the system was within specification during the test period and no additional uncertainty needs to be applied. 
Appendix A. Pictures of the Test Site 


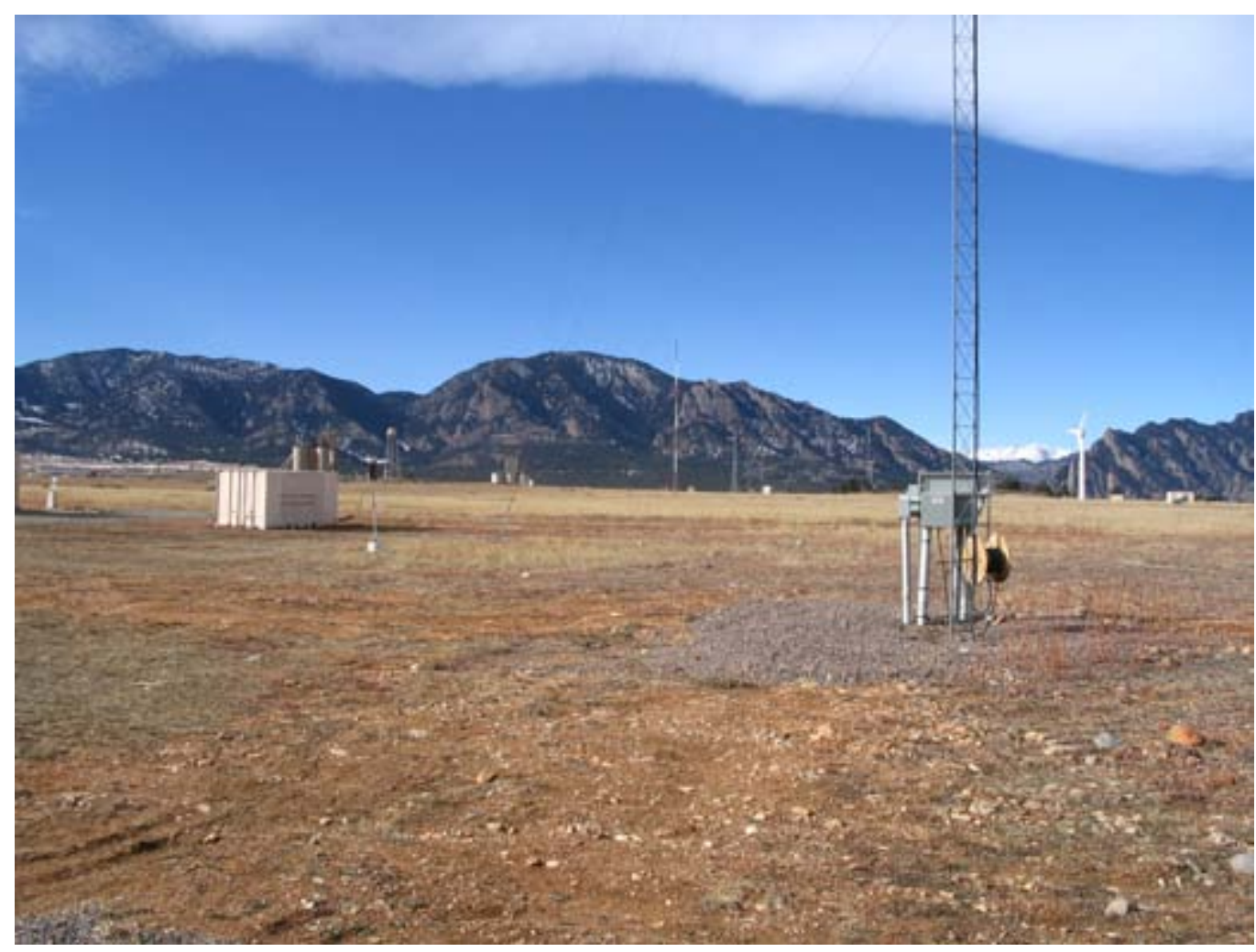

Figure A.1. West

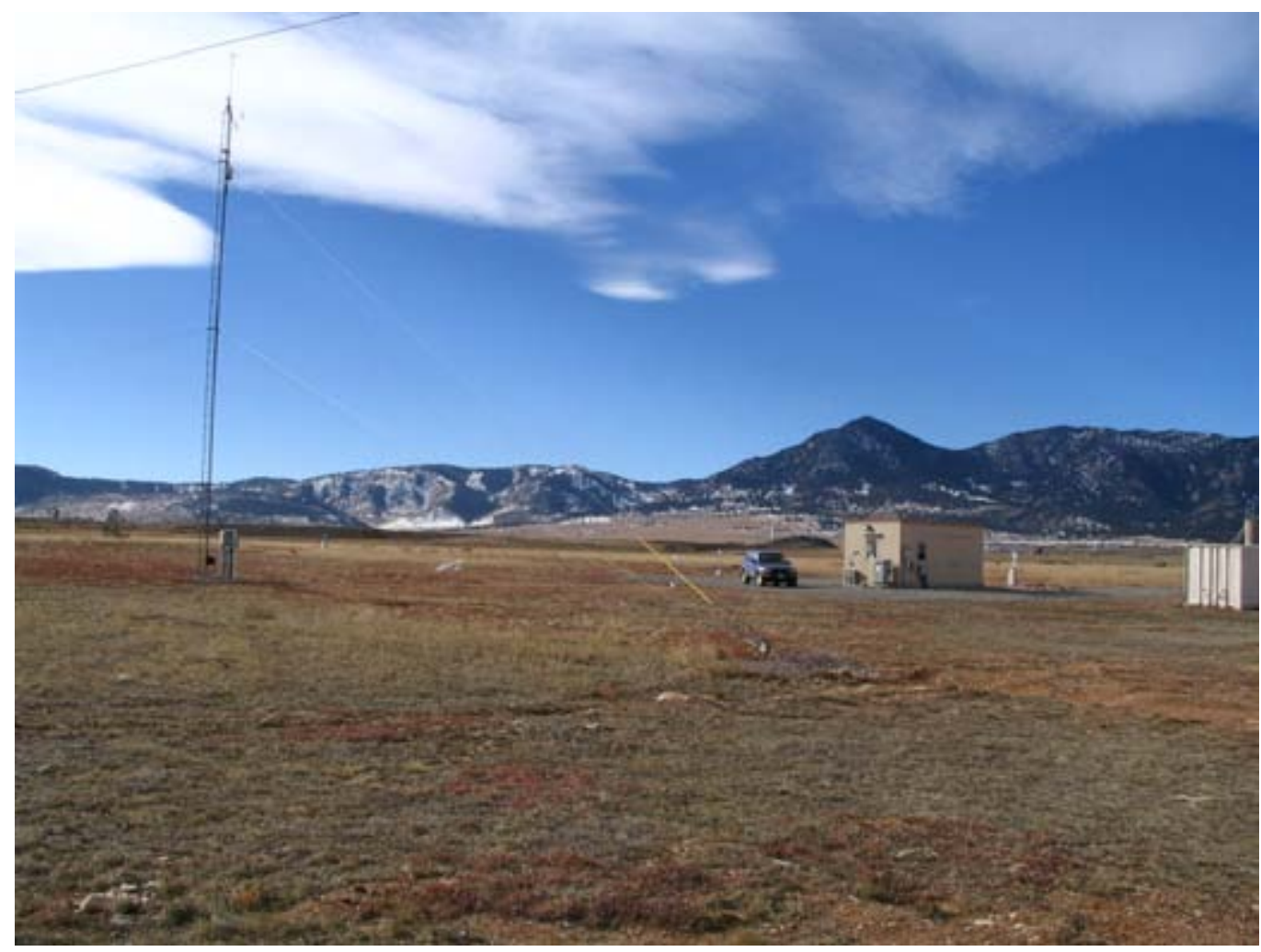

Figure A.2. Southwest 


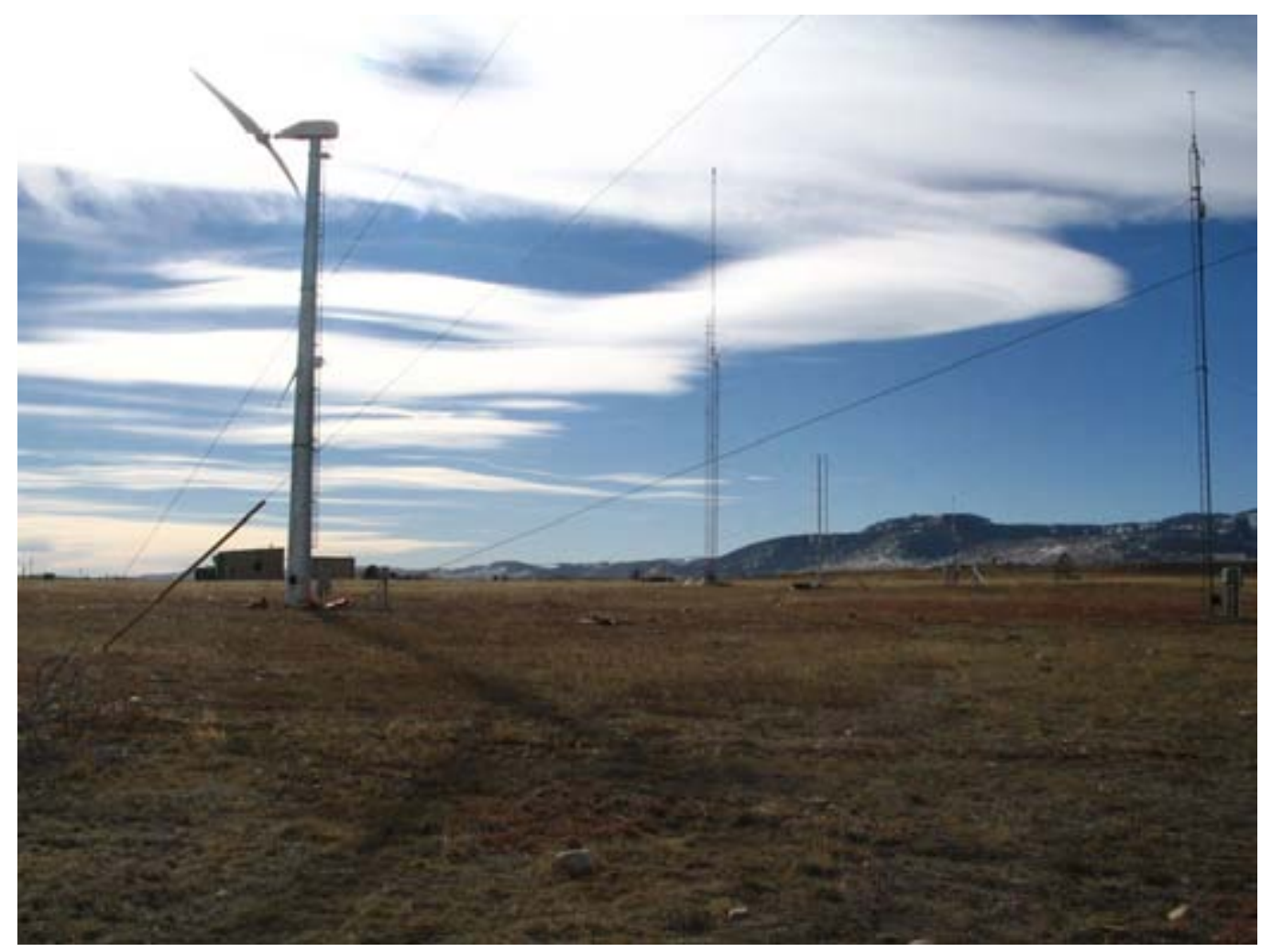

Figure A.3. South-south-west

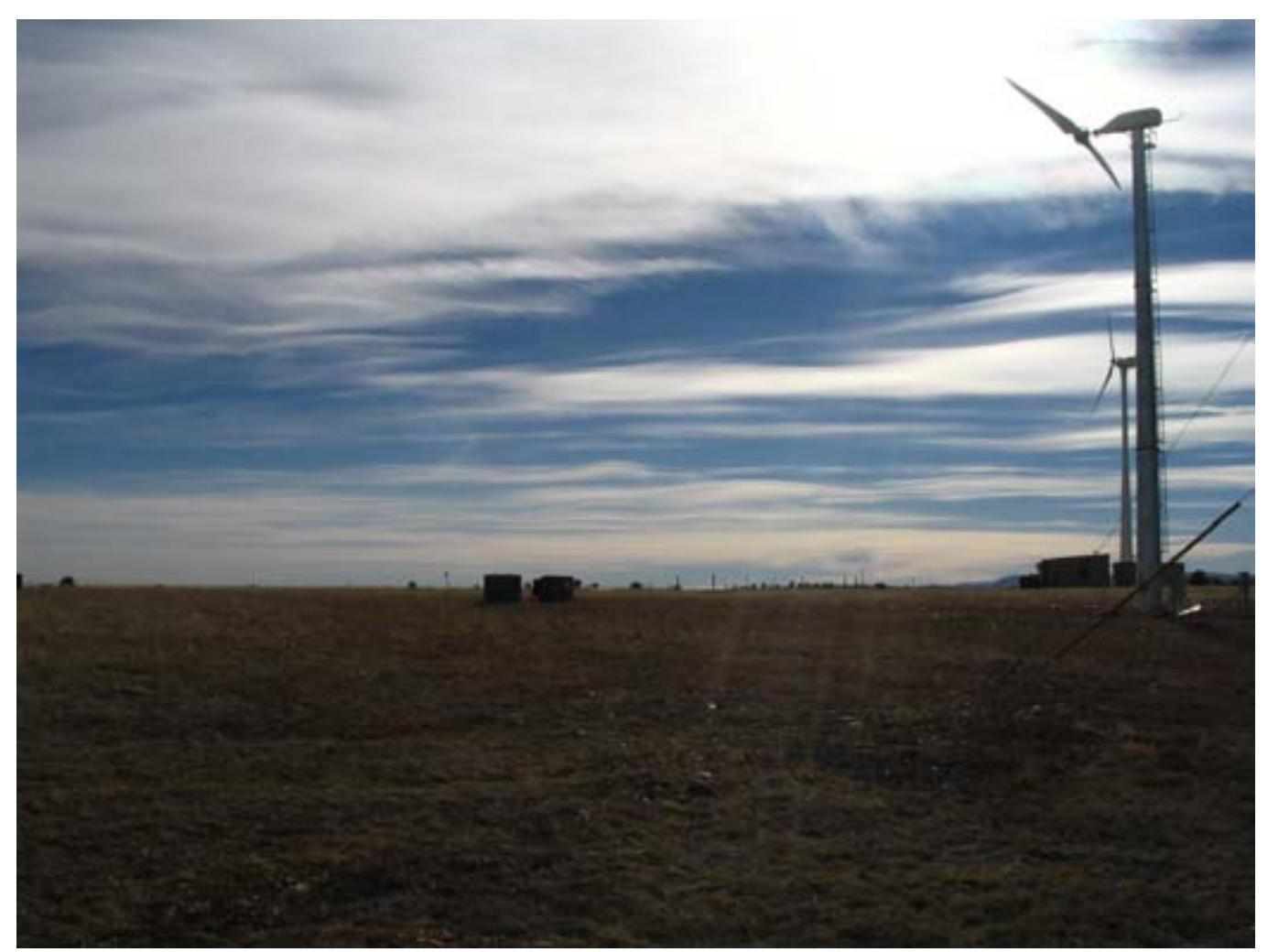

Figure A.4. South southeast 


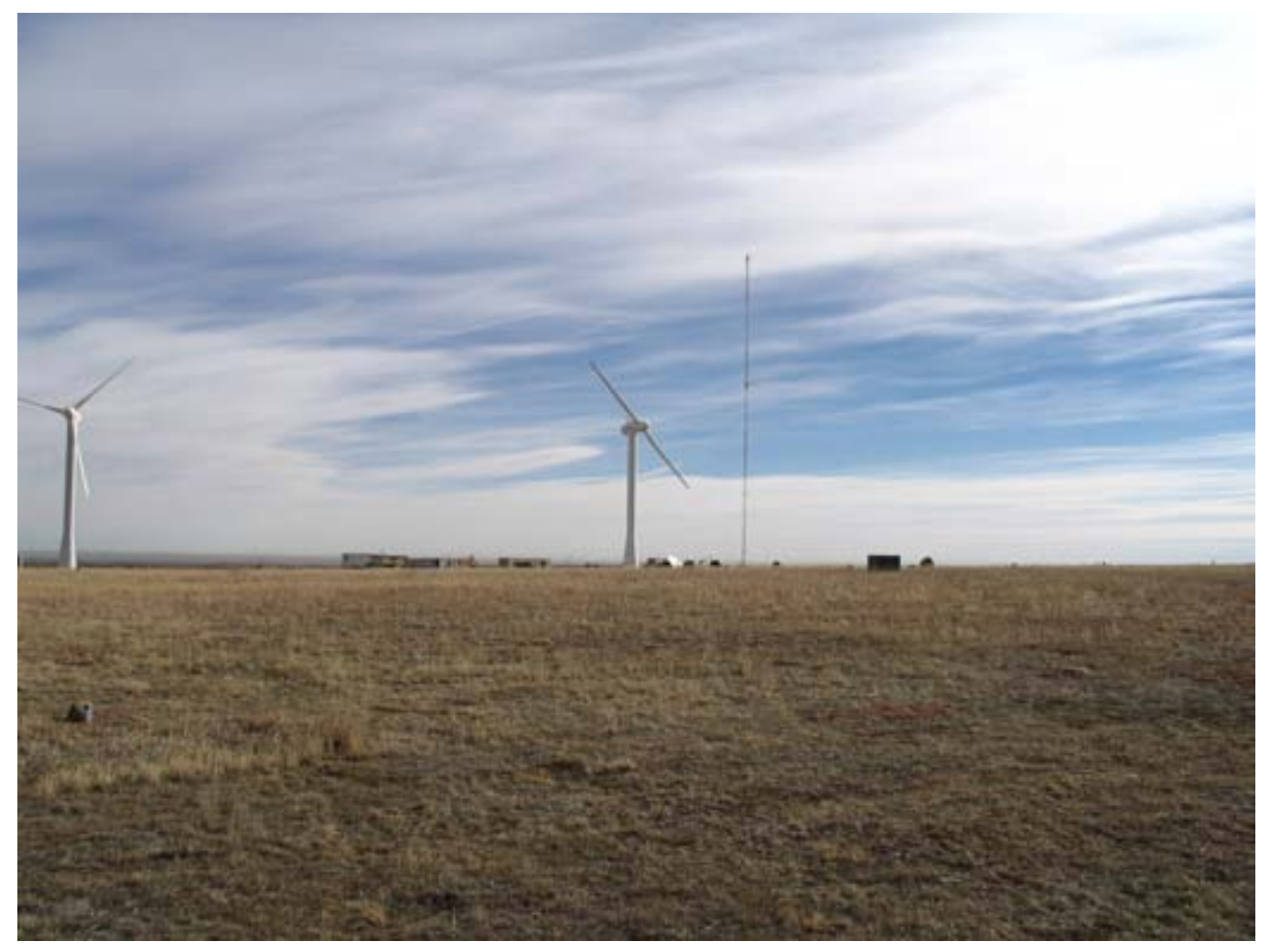

Figure A.5. East

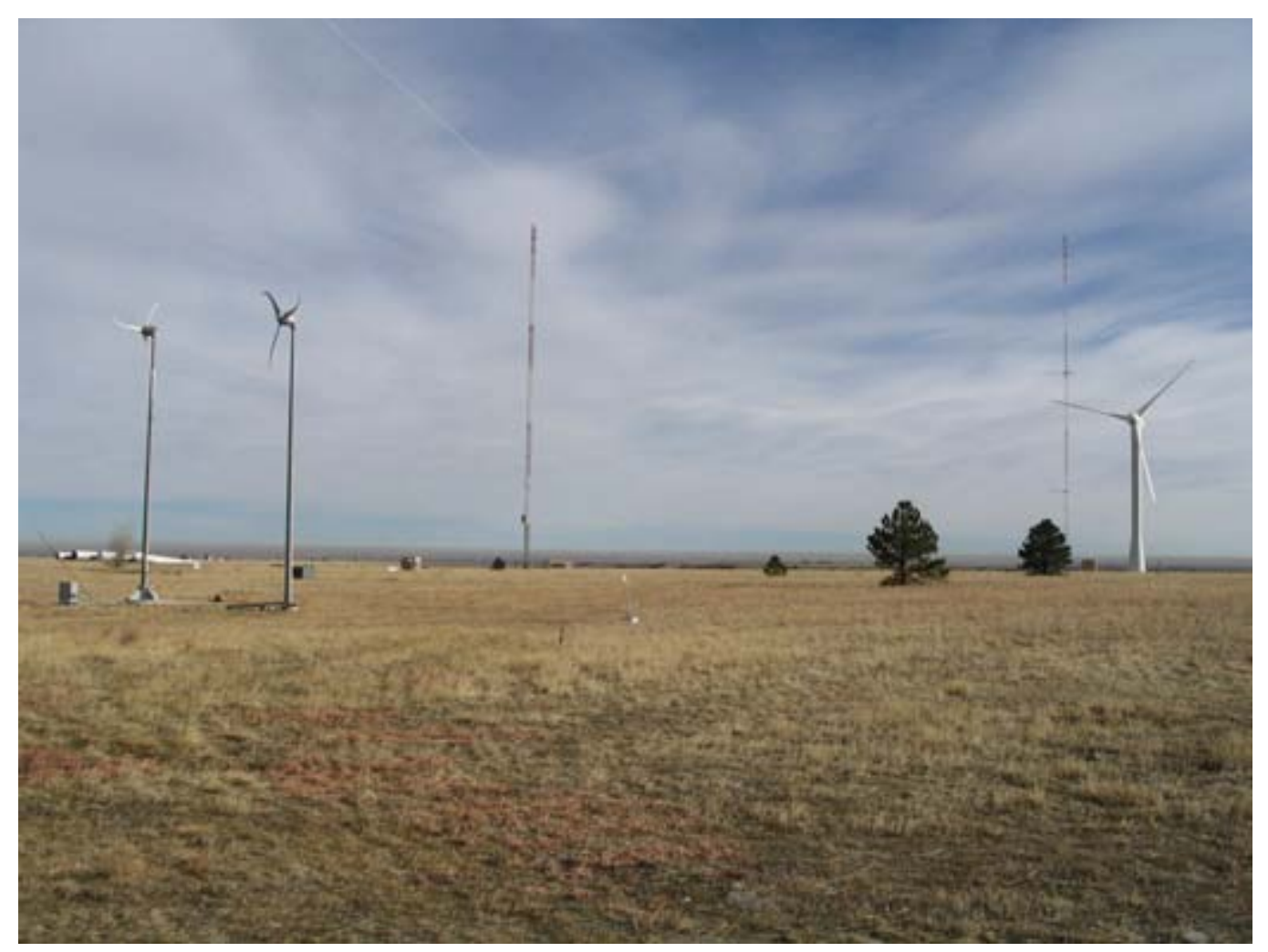

Figure A.6. Northeast 


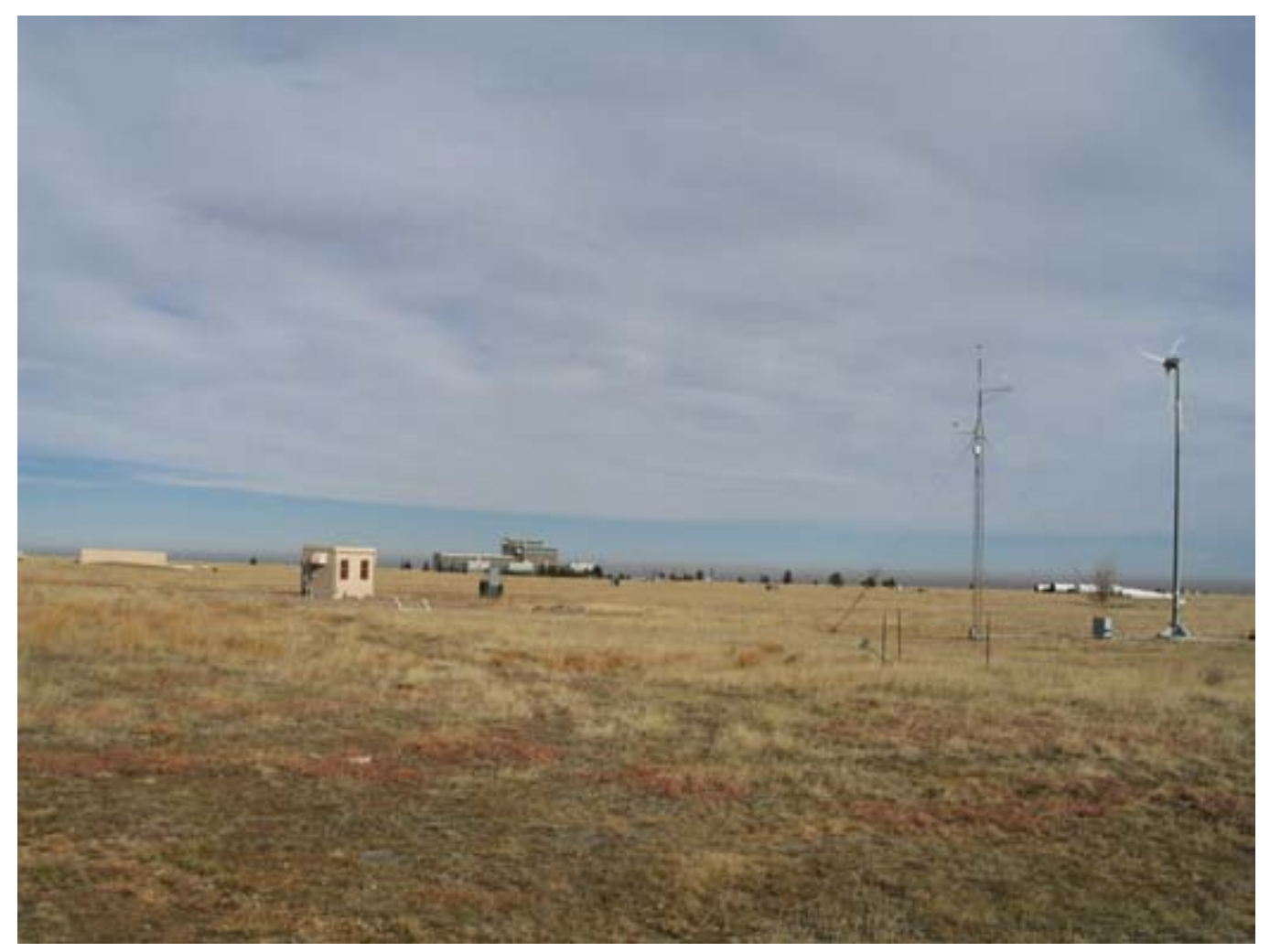

Figure A.7. North northeast

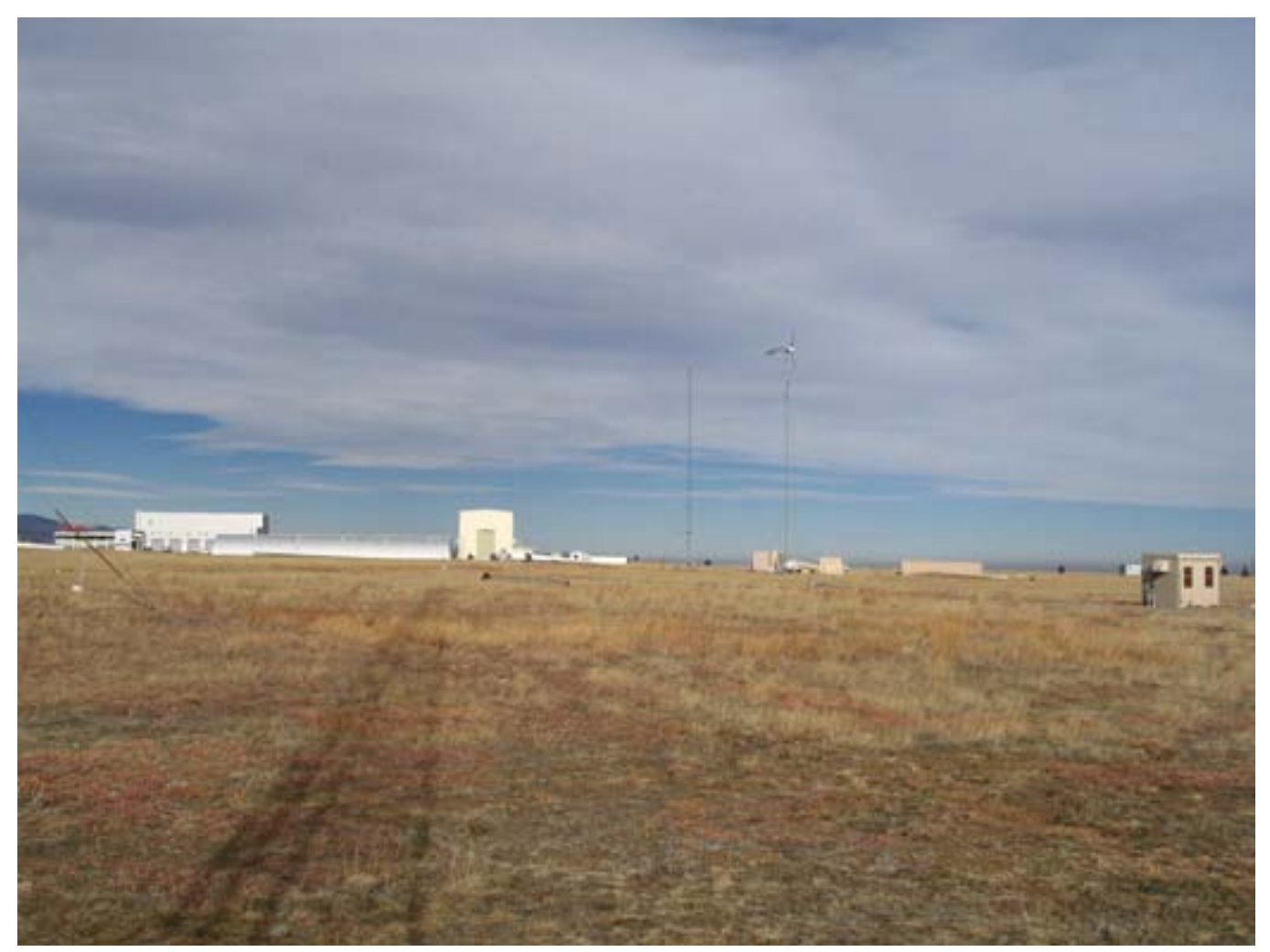

Figure A.8. North 


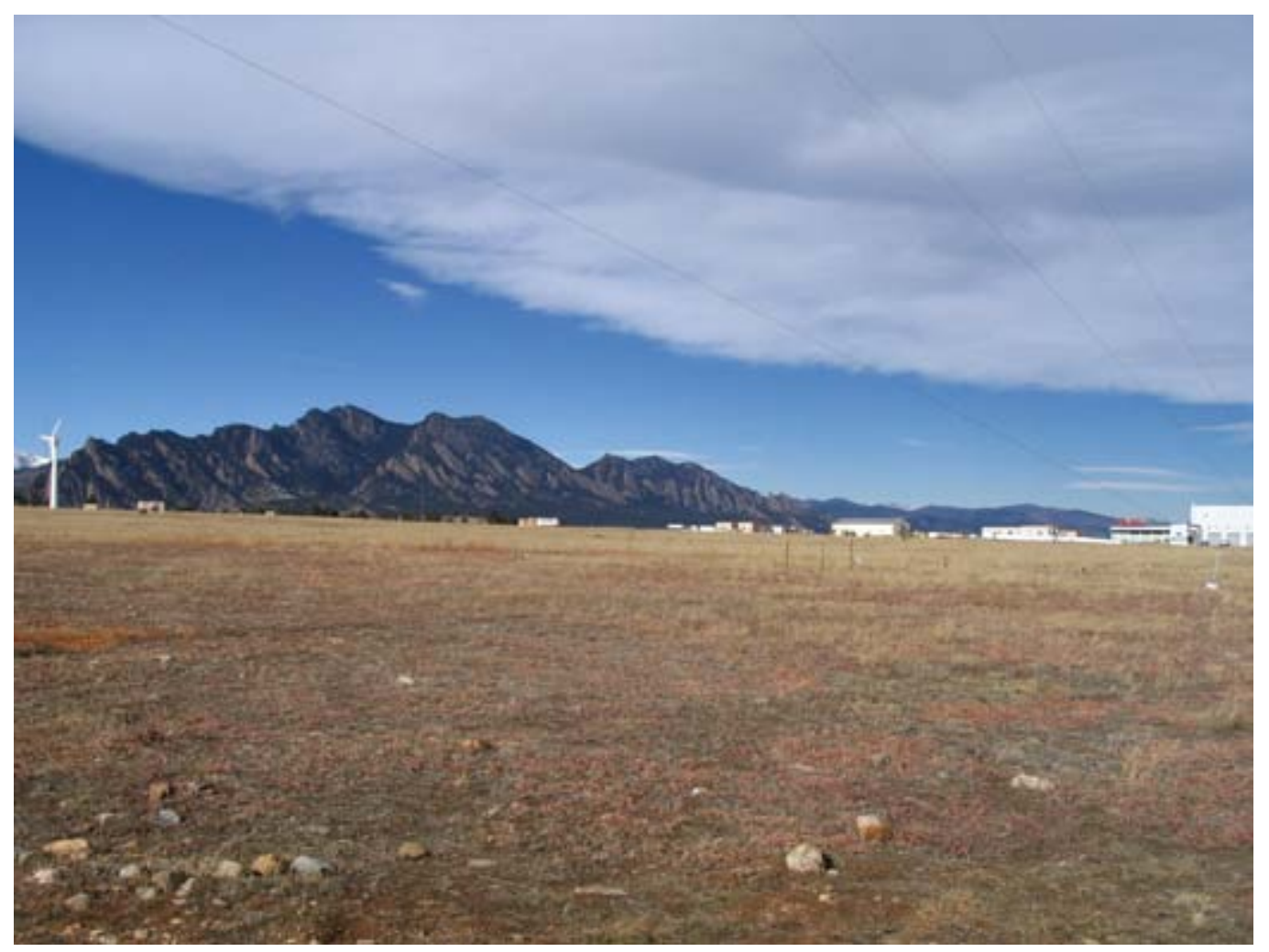

Figure A.9. Northwest 
Appendix B. Equipment Calibration Sheets 
Dranch \#2 5000

\section{NREL METROLOGY LABORATORY}

\section{Test Report}

Test Inetrument; Phaser Powor Transducex \& 2-CTs

DOE \# : $02824 C$

Model

t Phasex $-5-F-5 A$

$S / N \quad: 02663$

Calibxation Datez $01 / 28 / 2008$

Due Date: $01 / 28 / 2010$

A. Sot-UD for Total Real Powor Callbration:

A.1. Voltago $1 \mathrm{~s}$ applied to phases $A \& B=120 \mathrm{~V}$ a $60 \mathrm{~Hz}$.

A.2. Current is applied to $n=5$-TURNS through two current transformers

that are connected to phasos $A \& B$.

A.3. Analog Output-1 1 mensured across precision resistor $=250 \Omega$.

A.4. Phaser Fu11 Scale setting $=-7.2 \mathrm{KW}$ to $7.2 \mathrm{KW}$.

\begin{tabular}{|c|c|c|}
\hline $\begin{array}{c}\text { Input Current } \\
\text { (AAC) }\end{array}$ & $\begin{array}{c}\text { Input Power } \\
(K W)\end{array}$ & $\begin{array}{c}\text { Analog Output-1 } \\
\text { (VDC) }\end{array}$ \\
\hline 28 & 6.72 & 4.790 \\
\hline 21 & 5.04 & 4.341 \\
\hline 14 & 3.36 & 3.892 \\
\hline 7 & 1.68 & 3.444 \\
\hline 0 & 0 & 2.995 \\
\hline-7 & -1.68 & 2.547 \\
\hline-14 & -3.36 & 2.099 \\
\hline-21 & -5.04 & 1.651 \\
\hline-28 & -6.72 & 1.203 \\
\hline \hline
\end{tabular}

B. Set-UD for Power Factor Calibration:

B.1. Voltage $\&$ Current are applied as $A .1 \&$ A.2.

B.2. Analog Output-2 is measured across procision resistor $=250 \Omega$.

\begin{tabular}{|c|c|c|}
\hline $\begin{array}{c}\text { Power } \\
(\mathrm{KW})\end{array}$ & Power Factor & $\begin{array}{c}\text { Analog Output-2 } \\
\text { (VDC) }\end{array}$ \\
\hline 6.72 & 1.0 & 4.989 \\
\hline$"$ & 0.8 & 4.179 \\
\hline$n$ & 0.6 & 3.377 \\
\hline$n$ & 0.4 & 2.577 \\
\hline$n$ & 0.2 & 1.778 \\
\hline
\end{tabular}

Page 1 of 2

Figure B.1. Power transducer calibration sheet 


\section{DEUTSCHER KALIBRIERDIENST DKD}

Kalibrierlaboratorium für Stromungsgeschwindigkeit von Luft Calibration laboratory for velocity of air flow

Akkreditiert durch die / accredited by the

Akkreditierungsstelle des DKD bei der

PHYSIKALISCH-TECHNISCHEN BUNDESANSTALT (PTB)
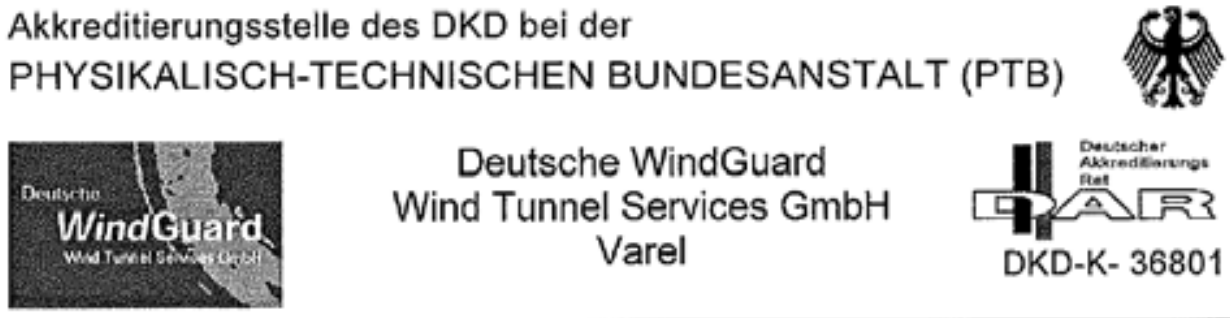

\section{Deutsche WindGuard \\ Wind Tunnel Services $\mathrm{GmbH}$ \\ Varel}

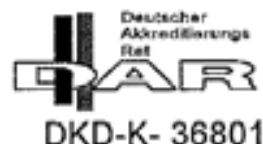

\section{Kalibrierschein \\ Calibration Certificate}

\begin{tabular}{|c|c|}
\hline $\begin{array}{l}\text { Gegenstand } \\
\text { Objuct }\end{array}$ & Cup Anemometer \\
\hline $\begin{array}{l}\text { Hersteller } \\
\text { Manuísctiver }\end{array}$ & $\begin{array}{l}\text { Thies Clima } \\
\text { D-37083 Gottingen }\end{array}$ \\
\hline Typ & 4.3350 .00 .000 \\
\hline $\begin{array}{l}\text { Fabrikat/Serien-Nr. } \\
\text { Sortai number }\end{array}$ & $\begin{array}{l}\text { Body: } 0707886 \\
\text { Cup: } 0707886\end{array}$ \\
\hline $\begin{array}{l}\text { Auftraggeber } \\
\text { Customir }\end{array}$ & $\begin{array}{l}\text { Thies Clima } \\
\text { D-37083 Gottingen }\end{array}$ \\
\hline $\begin{array}{l}\text { Auftragsnummer } \\
\text { Order } \mathrm{Na}\end{array}$ & VT07255 \\
\hline \multicolumn{2}{|c|}{$\begin{array}{l}\text { Anzahl der Seiten des Kal brierscheines } \\
\text { Wumber of pages of the cortificato }\end{array}$} \\
\hline $\begin{array}{l}\text { Datum der Kalibrierung } \\
\text { Oete of ceibretion }\end{array}$ & 24.07 .2007 \\
\hline
\end{tabular}

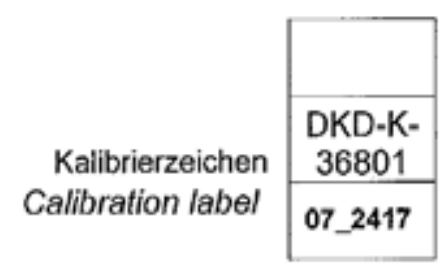

Dieser Kallbrlerschein dokumentiert die Rückfuhrung auf nationale Normale zur Darstellung der Einheiten in Obereinstimmung mit dem Internationalen Einheitensystem (SI). Der DKD ist Unterzeichner der multi- lateralen Obereinkommen der European co-operation for Accreditation (EA) und der International Laboratory Accreditation Cooperation (ILAC) zur gegenseitigen Anerkennung der Kalibrierscheine.

Fôr die Einhaltung einer angemessenen frist zur Wiederholung der Kalibrierung ist der Benutzer verantwortlich

This calibration certificate documonts the traceability to national standards, which realize the units of measurement according to the Intemational System of Unils (Sl).

The DKD is signatory to the muitiateral agreements of the European co-operation for Accroditation (EA) and of the intemational Laboratory Accreditation Cooperotion (ILAC) for the mutual recognition of calibration certificates.

The user is obliged to have the objoct recalibrated at appropriate intervals.

Dieser Kalibrierschein darf nur vollständig und unverändert weiterverbreitet werden. Auszüge oder Ãnderungen bedorfen der Genehmigung sowohl der Akkreditierungsstelle des DKD als auch des ausstellenden Kalibrierlaboratoriums. Kalibrierscheine ohne Unterschrift und Stempel haben keine Guttigkeit.

This cafibration certificate may nof be reproduced other than in full except with the pormission of both the Accreditation Body of the DKD and the lssuing laboratory. CaNbration certificates without signature and seal are not valid.

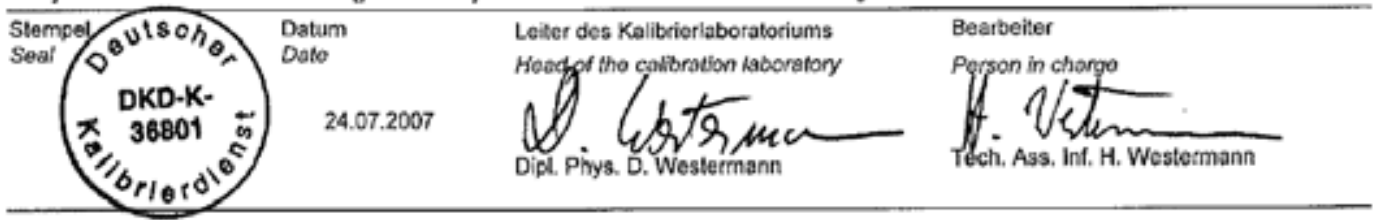

Deutsche WindGuard Wind Tunnet Services GmbH Oldenburger Str. 65

26316 Varel ; Tel. ++49 (0)4451 95150

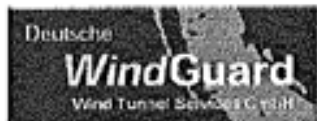

Figure B.2. Primary anemometer calibration sheet I 


\section{DEUTSCHER KALIBRIERDIENST DKD}

Kalibrierlaboratorium for Strömungsgeschwindigkeit von Luft

Calibration laboratory for veiacity of air flow

Akkreditiert durch die / accredited by the

Akkreditierungsstelle des Deutschen Kalibrierdienstes

DEWI

\section{Kalibrierschein \\ Calibration certificate}

\section{DEWI GmbH \\ Deutsches Windenergie-Institut}

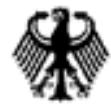

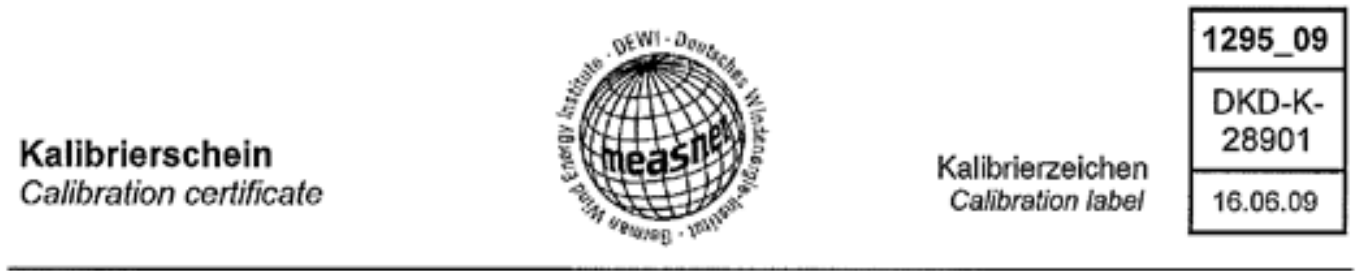

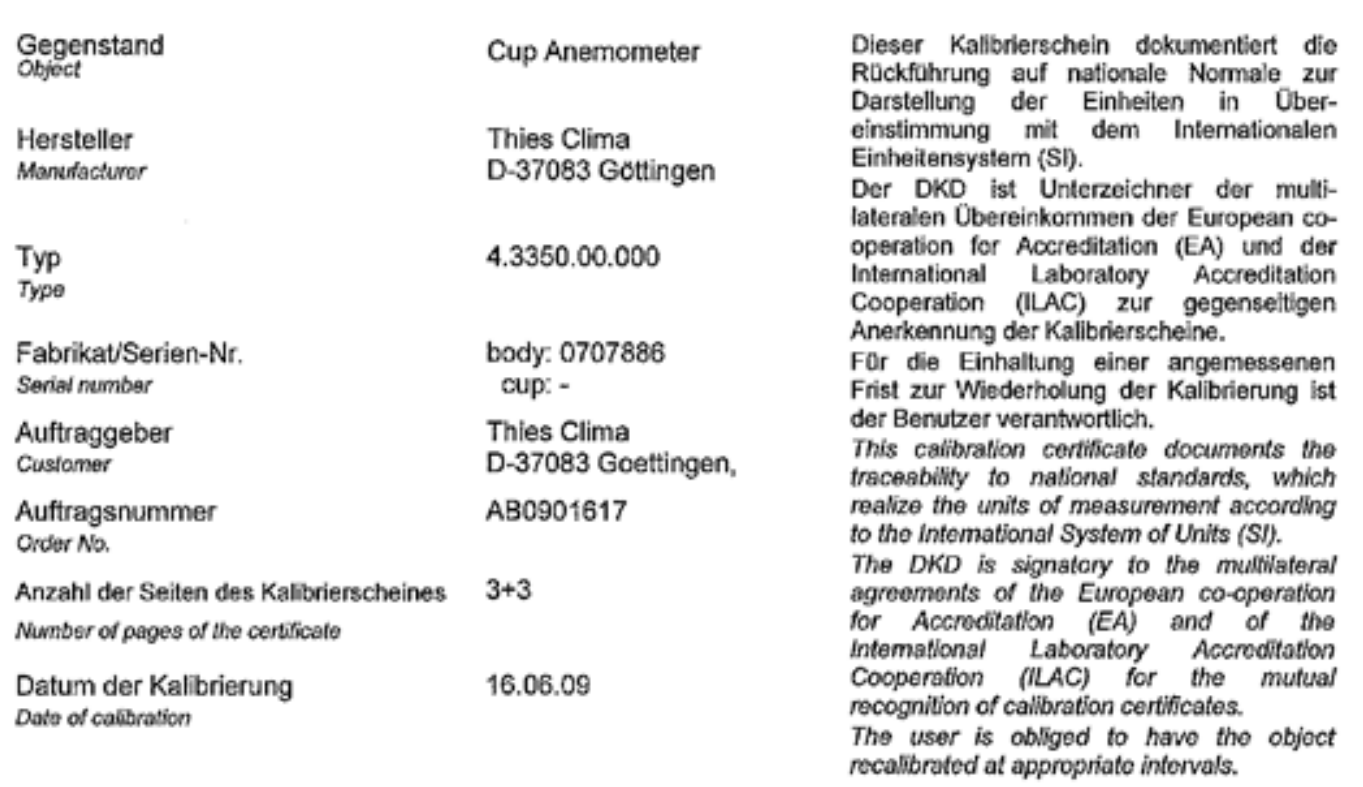

Dieser Kalbrierschein darf nur vollständig und unverändert weiterverbreitet werden. Auszüge oder Ånderungen bedürfen der Genehmigung sowohl der Akkreditierungsstele des DKD als auch des ausstellenden Kalibrierlaboratoriums. Kalibrierscheine ohne Unterschrift und Stempel haben keine Gultigkeit.

This calibration certificate may not be reproduced other than in full except with the permission of both the Accreditation Body of the DKD and the issuing laboratory. Calibration certificates without slgnature and sool are not valid.

\begin{tabular}{|c|c|c|c|}
\hline$\left(\begin{array}{c}\text { DKD-K- } \\
28901 \\
{ }^{2}\end{array}\right.$ & $\begin{array}{l}\text { Datum } \\
\text { Dote }\end{array}$ & $\begin{array}{l}\text { Stelliv. Leiler des Ka kriarlabcratoriums } \\
\text { Doputy head of tho caibration loboratory }\end{array}$ & $\begin{array}{l}\text { Bearbeiter } \\
\text { Person in chargo }\end{array}$ \\
\hline
\end{tabular}

DEWI GMbH DEUTSCHES WINDENERGIE - INSTITUT

Ebertstr. 96, D-26382 Wilhelmshaven

Tel. +49 (0) $44214808-0$, Fax. $+49(0) 44214808-43$

Figure B.3. Primary anemometer calibration sheet II 


\section{Wind Vane Calibration Report}

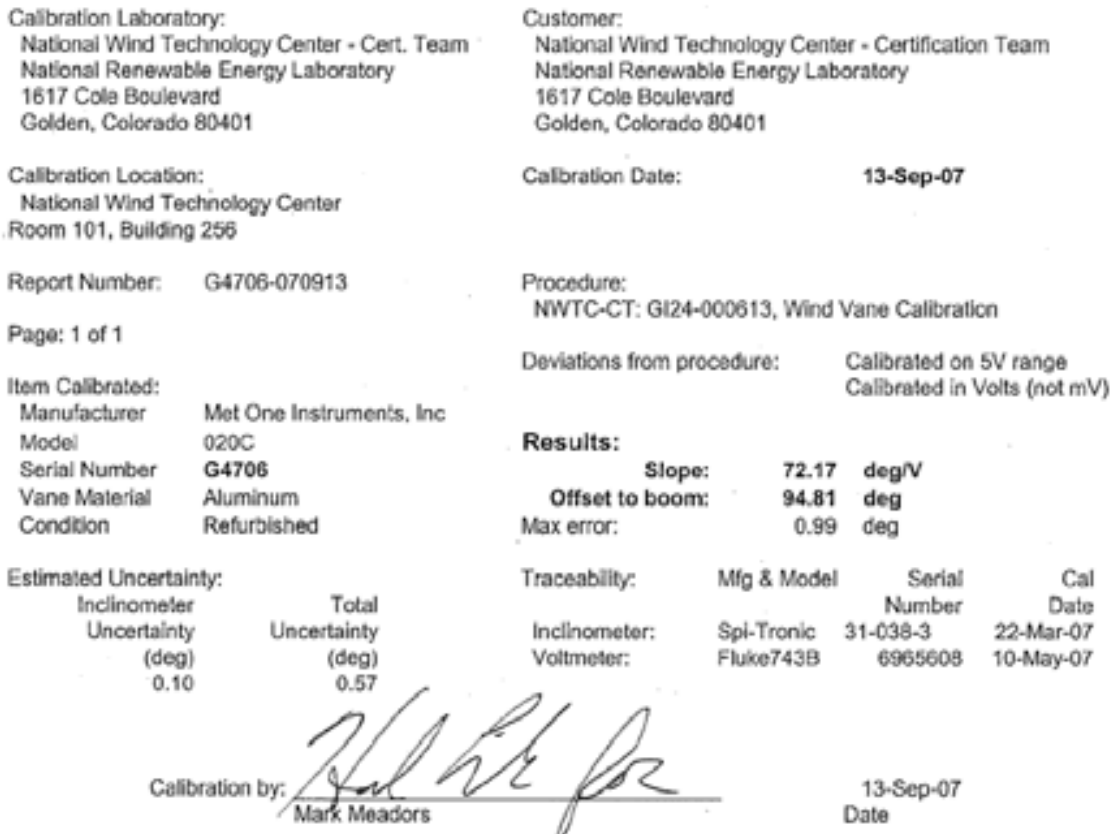

Results:

Offset to boom: $\quad 94.81$ deg

Max error:

$0.99 \mathrm{deg}$

13-Sep-07

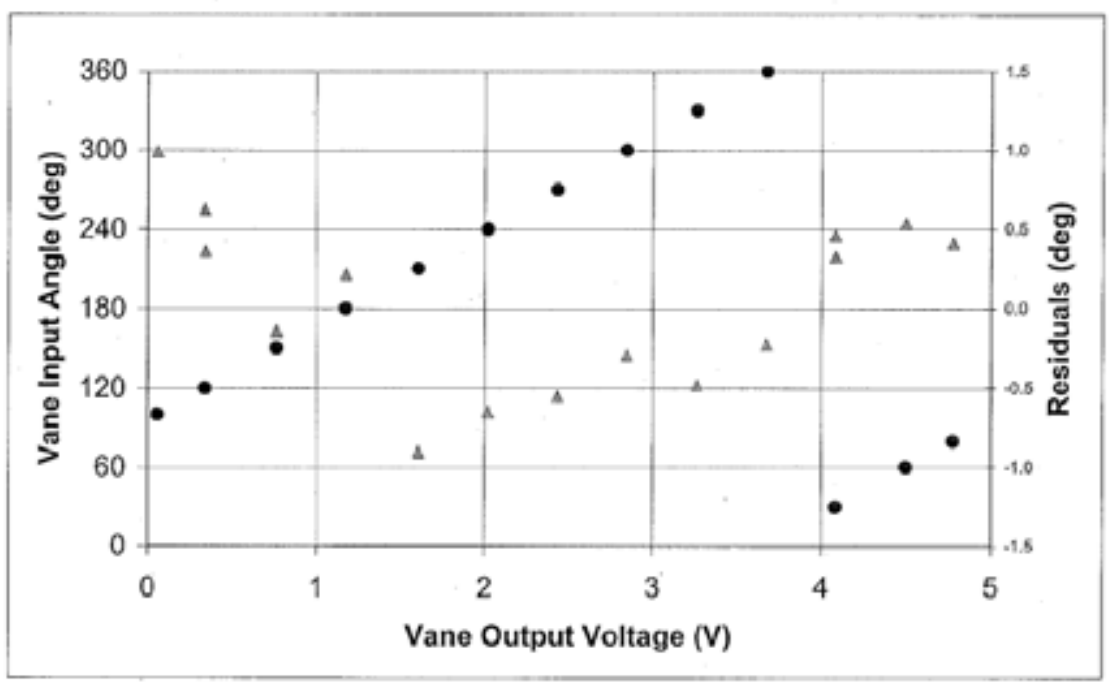

Figure B.4. Wind vane calibration report 
NREL METROLOGY LABORATORY

Test Report

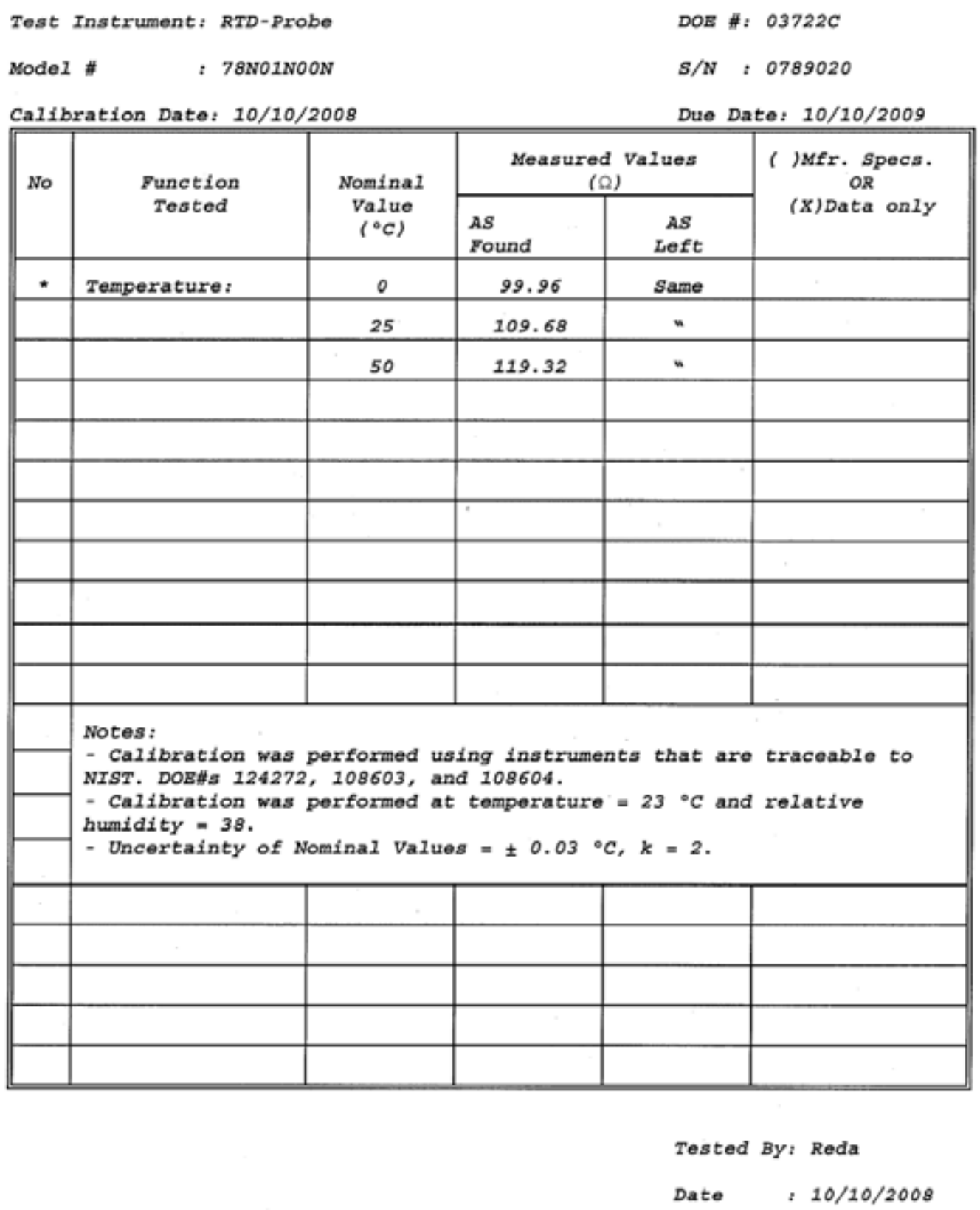

Figure B.5. RTD-Probe calibration sheet 


\section{NREL METROLOGY LABORATORY}

Test Report

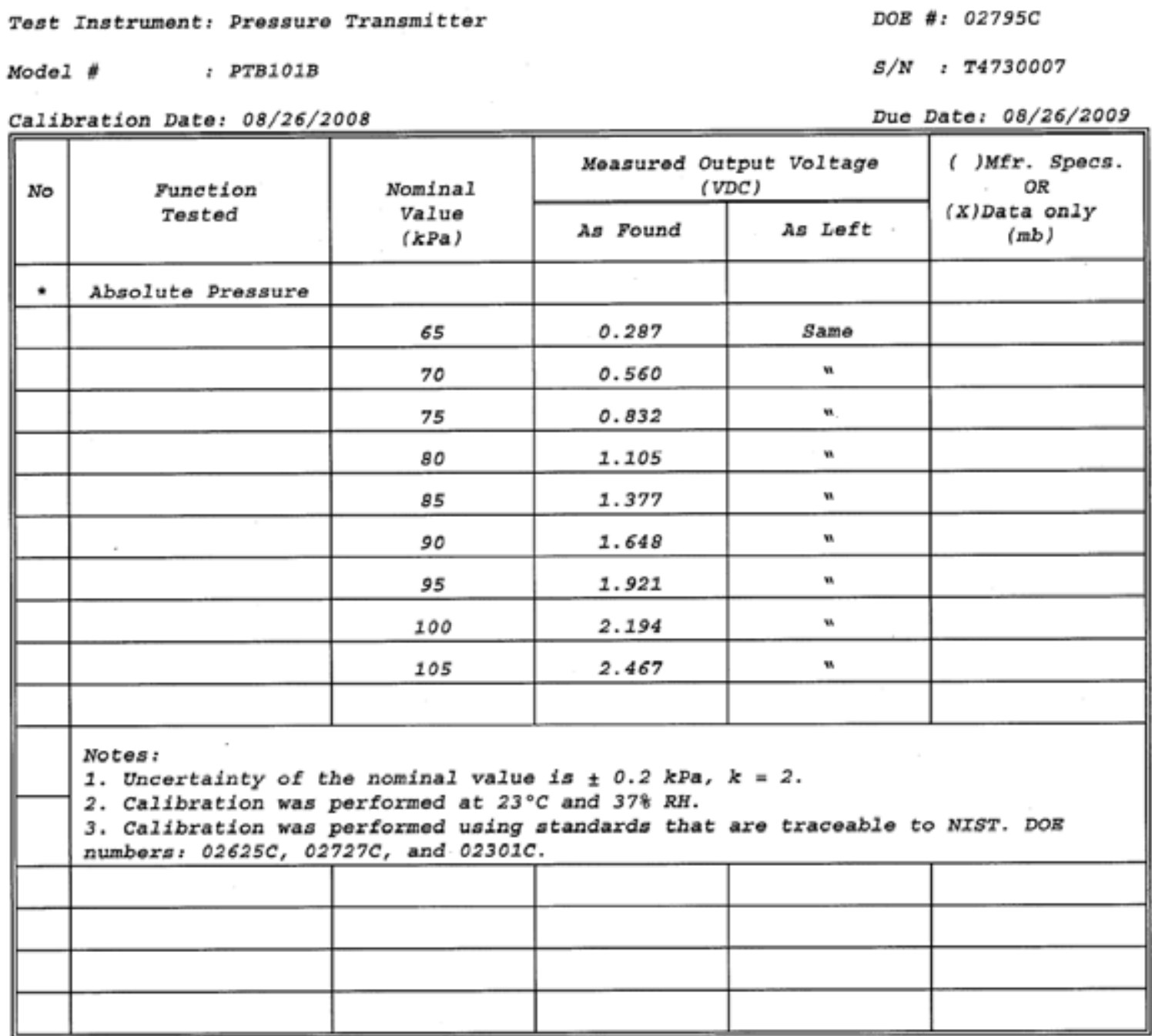

Figure B.6. Pressure transmitter calibration sheet 
Board Information:

Serial Number: 12C73B4

NI Part Number: 192547D-01

Description: NI 9217

Calibration Date: 03-AUG-07

Recommended Calibration Due Date: 03-AUG-08*

Ambient Temperature: $23{ }^{\circ} \mathrm{C}$

Relative Humidity: $46 \%$
Certificate Information:

Certificate Number: 786529

Date Printed: 05-JAN-09

National Instruments certifies that at the time of manufacture, the above product was calibrated in accordance with applicable National Instruments procedures. These procedures are in compliance with relevant clauses of ISO 9001 and are designed to assure that the product listed above meets or exceeds National Instruments specifications.

National Instruments further certifies that the measurements standards and instruments used during the calibration of this product are traceable to National and/or International Standards administered by NIST or Euromet members or are derived from accepted values of natural physical constants.

The environment in which this product was calibrated is maintained within the operating specifications of the instrument and the standards.

The information shown on this certificate applies only to the instrument identified above and the certificate may not be reproduced, except in full, without prior written consent by National Instruments.

For questions or comments, please contact National Instruments Technical Support.

NI Hungary Software és Hardware Gyártó Kft. 4031 Debrecen, Határ út $1 / A$.

HUNGARY

* Recommended calibration due date is based on a combination of calibration interval and, when applicable, calibration shelf life. This date may vary depending on your application requirements.

Figure B.7. NI 9217 data acquisition module calibration sheet I 
Board Information:

Serial Number: 12A2037

NI Part Number: 192580D-02

Description: NI 9229

Calibration Date: 31-MAY-07

Recommended Calibration Due Date: 31-MAY-08*

Ambient Temperature: $22{ }^{\circ} \mathrm{C}$

Relative Humidity: $50 \%$
Certificate Information:

Certificate Number: 733748

Date Printed: 05-JAN-09

National Instruments certifies that at the time of mamufacture, the above product was calibrated in accordance with applicable National Instruments procedures. These procedures are in compliance with relevant clauses of ISO 9001 and are designed to assure that the product listed above meets or exceeds National Instruments specifications.

National Instruments further certifies that the measurements standards and instruments used during the calibration of this product are traceable to National and/or International Standards administered by NIST or Euromet members or are derived from accepted values of natural physical constants.

The environment in which this product was calibrated is maintained within the operating specifications of the instrument and the standards.

The information shown on this certificate applies only to the instrument identified above and the certificate may not be reproduced, except in full, without prior written consent by National Instruments.

For questions or comments, please contact National Instruments Technical Support.

NI Hungary Software és

Hardware Gyártó Kft.

4031 Debrecen, Határ út

$1 / A$.

HUNGARY
Signed,

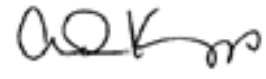

Andrew Krupp Quality Director

* Recommended calibration due date is based on a combination of calibration interval and, when applicable, calibration shelf life. This date may vary depending on your application requirements.

Figure B.8. NI 9229 data acquisition module calibration sheet I 
Board Information:

Serial Number: 12ECB77

NI Part Number: 193299F-01

Description: NI-9205

Calibration Date: 09-OCT-07

Recommended Calibration Due Date: 09-OCT-08*

Ambient Temperature: $23^{\circ} \mathrm{C}$

Relative Humidity: $37 \%$
Certificate Information:

Certificate Number: 837236

Date Printed: 05-JAN-09

National Instruments certifies that at the time of manufacture, the above product was calibrated in accordance with applicable National Instruments procedures. These procedures are in compliance with relevant clauses of ISO 9001 and are designed to assure that the product listed above meets or exceeds National Instruments specifications.

National Instruments further certifies that the measurements standards and instruments used during the calibration of this product are traceable to National and/or International Standards administered by NIST or Euromet members or are derived from accepted values of natural physical constants.

The environment in which this product was calibrated is maintained within the operating specifications of the instrument and the standards.

The information shown on this certificate applies only to the instrument identified above and the certificate may not be reproduced, except in full, without prior written consent by National Instruments.

For questions or comments, please contact National Instruments Technical Support.

NI Hungary Software és

Hardware Gyártó $K f t$.

4031 Debrecen, Határ út

$1 / A$.

HUNGARY
Signed,

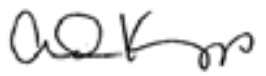

Andrew Krupp

Quality Director

* Recommended calibration due date is based on a combination of calibration interval and, when applicable, calibration shelf life. This date may vary depending on your application requirements.

Figure B.9. NI 9205 data acquisition module calibration sheet I 


\section{Davis/Calibration}

Company ID: 229037

NATIONAL INSTRUMENTS

11500 N. MOPAC EXPWY

ATTN. RMA DEPT.

AUSTIN, TX 78759

Instrument ID: $12 \mathrm{~A} 2037$

Manufacturer: NATIONAL INSTRUMENTS

Description: 4-CHANNEL, \pm 60 V, $24-B I T$ SIMULTANEOUS ANALOG INPUT

Instrument Identification

\section{Certificate of Calibration}

3214191

CALIBRATION

Certificate Page 1 of 1

PO Number: 337683

Model Number: NI 9229

Serial Number: 12A2037

Accuracy: Mfr Specifications

Certificate Information

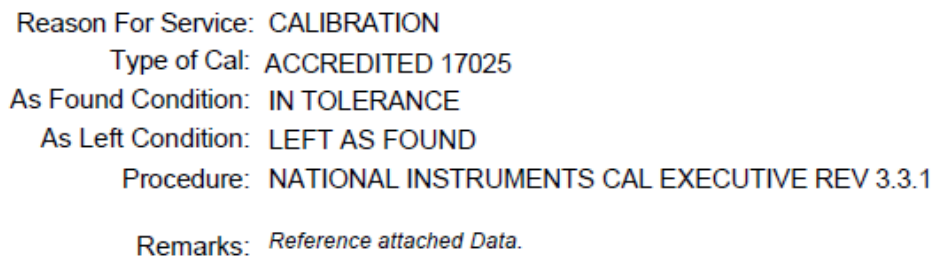

Technician: WAYNE GETCHELL Cal Date 06May2009

Cal Due Date: 06May2010 Interval: 12 MONTHS

Temperature: $23.0 \mathrm{C}$ Humidity: $44.0 \%$

\begin{tabular}{|c|c|c|c|c|c|}
\hline NIST Traceable\# & Inst. ID\# & Description & Model & Cal Date & Date Due \\
\hline 3143038 & $15-0271$ & MULTIFUNCTION CALIBRATOR & $5700 \mathrm{~A}$ & 15Apr20 & 14Jul2009 \\
\hline
\end{tabular}

Davis Calibration • 2324 Ridgepoint Drive, Suite D • Austin, TX $78754 \cdot$ Phone: 800-365-0147 • Fax: 512-926-8450

Figure B.10. NI 9229 data acquisition module calibration sheet II 


\section{Davis|Calibration $\Lambda$}

Company ID: 229037

NATIONAL INSTRUMENTS

11500 N. MOPAC EXPWY

ATTN. RMA DEPT.

AUSTIN, TX 78759

Instrument ID: 12C73B4

Manufacturer: NATIONAL INSTRUMENTS

Description: 4-CH 100 OHM 24-BIT RTD ANALOG INPUT

Accuracy: Mfr. Specifications

\section{Certificate of Calibration}

3214178

Certificate Page 1 of 1

Instrument Identification

PO Number: 337683

Model Number: NI 9217

Serial Number: 12C73B4

Certificate Information

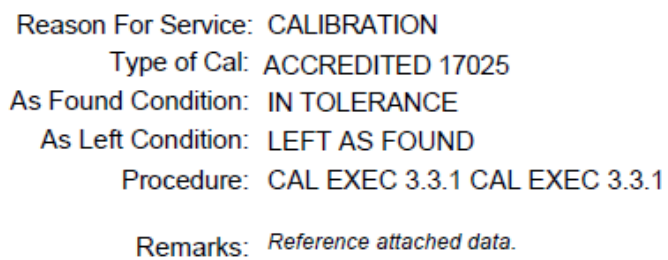

Technician: WAYNE GETCHELL Cal Date 06May2009 Cal Due Date: 06May2010 Interval: 12 MONTHS

Temperature: $23.0 \mathrm{C}$ Humidity: $46.0 \%$

Approved By: VICTOR PENA

Service Representative

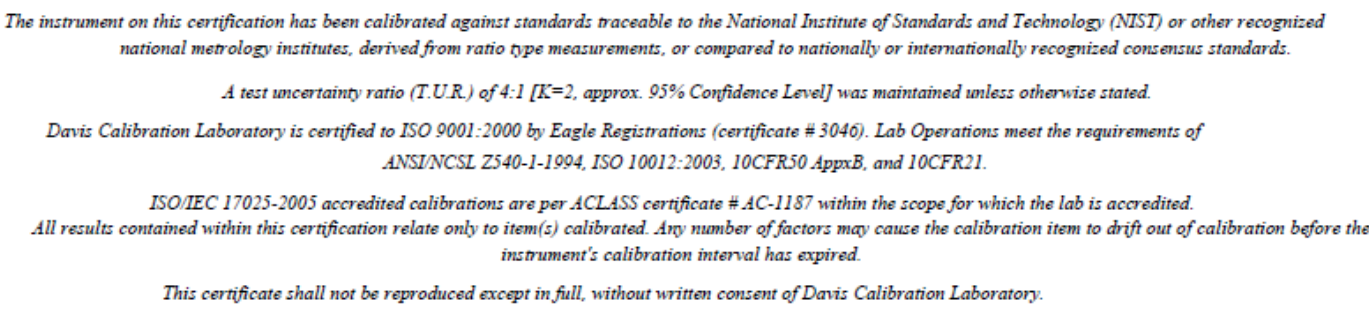

This certificate shall not be reproduced except in full, without written consent of Davis Calibration Laboraton.

\section{Calibration Standards}

\begin{tabular}{|c|c|c|c|c|c|}
\hline NIST Traceable\# & Inst. ID\# & Description & Model & Cal Date & Date Due \\
\hline 3078982 & $15-0011$ & DECADE RESISTOR & DB52 & 24Mar2009 & 24Mar2010 \\
\hline 3004176 & $15-0060$ & DIGITAL MULTIMETER (GOLDEN CAL) & 3458A OPT 002 & 17Feb2009 & 17May2009 \\
\hline
\end{tabular}

Davis Calibration • 2324 Ridgepoint Drive, Suite D • Austin, TX 78754 •Phone: 800-365-0147 • Fax: 512-926-8450

Figure B.11. NI 9217 data acquisition module calibration sheet II 


\section{Davis Calibration}

Company ID: 229037

NATIONAL INSTRUMENTS

11500 N. MOPAC EXPWY

ATTN. RMA DEPT.

AUSTIN, TX 78759

Instrument ID: 12ECB77

Manufacturer: NATIONAL INSTRUMENTS

Description: $32-\mathrm{CH} \pm 200 \mathrm{MV}$ TO $\pm 10 \mathrm{~V}, 16$-BIT, 250 KS/S ANALOG INPUT MODULE
PO Number: 337683
Instrument Identification

\section{Certificate of Calibration}

3214150

Certificate Page 1 of 1
Model Number: NI 9205

Serial Number: 12ECB77

Accuracy: Mfr Specifications

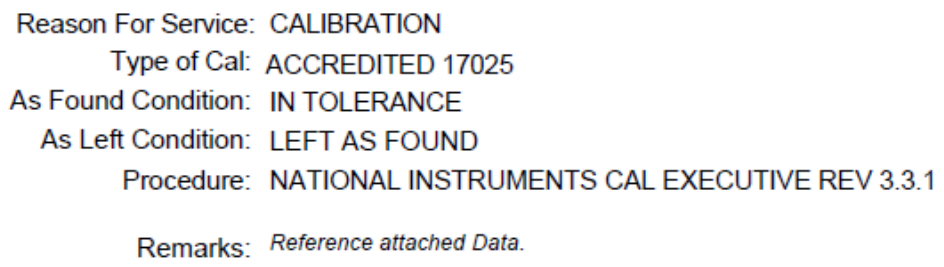

\author{
Technician: WAYNE GETCHELL \\ Cal Date 06May2009 \\ Cal Due Date: 06May2010 \\ Interval: 12 MONTHS \\ Temperature: $23.0 \mathrm{C}$ \\ Humidity: $47.0 \%$
}

\begin{abstract}
The instrument on this certification has been calibrated against standards traceable to the National Institute of Standards and Technology (NIST) or other recognized national metrology institutes, derived from ratio type measurements, or compared to nationally or intemationally recognized consensus standards.

Davis Calibration Laboratory is certified to ISO 9001:2000 by Eagle Registrations (certificate \#3040). Lab Operations meet the requirements of ANSINCSL Z540-1-1994, ISO 10012:2003, 10CFR50 AppxB, and 10CFR21.

ISOIIEC 17025-2005 accredited calibrations are per ACLASS certificate \#AC-1187 within the scope for which the lab is accredited. All results contained within this certification relate only to item(s) calibrated. Any number of factors may cause the calibration item to drift out of calibration before the instrument's calibration interval has expired.

This certificate shall not be reproduced except in fill, without written consent of Davis Calibration Laboratory.
\end{abstract}

Approved By: VICTOR PENA

Service Representative

\section{Calibration Standards}

$\frac{\text { NIST Traceable\# }}{3143038} \frac{\text { Inst. ID\# }}{15-0271} \quad \frac{\text { Description }}{\text { MULTIFUNCTION CALIBRATOR }} \quad \frac{\text { Model }}{5700 \mathrm{~A}} \frac{\text { Cal Date }}{15 \text { Apr2009 }}$

Figure B.12. NI 9205 data acquisition module calibration sheet II 


\section{REPORT DOCUMENTATION PAGE}

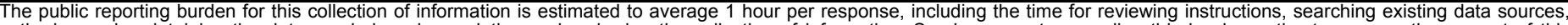

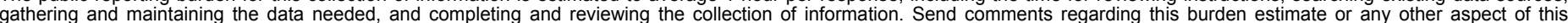

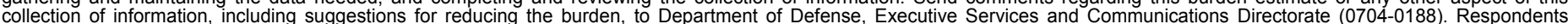

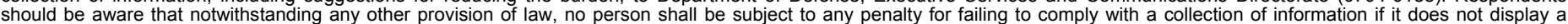

should be aware that notwithstanding

PLEASE DO NOT RETURN YOUR FORM TO THE ABOVE ORGANIZATION.

\begin{tabular}{l|l|l|}
\hline 1. REPORT DATE (DD-MM-YYYY) & 2. REPORT TYPE & 3. DATES COVERED (FrOm - TO)
\end{tabular}

February 2010

4. TITLE AND SUBTITLE

Wind Turbine Generator System - Power Performance Test Report:

ARE442 Wind Turbine

5a. CONTRACT NUMBER

DE-AC36-08-GO28308

5b. GRANT NUMBER

5c. PROGRAM ELEMENT NUMBER

6. AUTHOR(S)

J. van Dam and D. Jager 5d. PROJECT NUMBER

NREL/TP-500-46191

5e. TASK NUMBER

WE102211

5f. WORK UNIT NUMBER
7. PERFORMING ORGANIZATION NAME(S) AND ADDRESS(ES)

National Renewable Energy Laboratory

1617 Cole Blvd.

Golden, CO 80401-3393
8. PERFORMING ORGANIZATION REPORT NUMBER

NREL/TP-500-46191

9. SPONSORING/MONITORING AGENCY NAME(S) AND ADDRESS(ES)

10. SPONSOR/MONITOR'S ACRONYM(S)

NREL

11. SPONSORING/MONITORING AGENCY REPORT NUMBER

12. DISTRIBUTION AVAILABILITY STATEMENT

National Technical Information Service

U.S. Department of Commerce

5285 Port Royal Road

Springfield, VA 22161

13. SUPPLEMENTARY NOTES

14. ABSTRACT (Maximum 200 Words)

This report summarizes the results of a power performance test that NREL conducted on the ARE 442 wind turbine.

This test was conducted in accordance with the International Electrotechnical Commission's (IEC) standard, Wind

Turbine Generator Systems Part 12: Power Performance Measurements of Electricity Producing Wind Turbines, IEC 61400-12-1 Ed.1.0, 2005-12. However, because the ARE 442 is a small turbine as defined by IEC, NREL also followed Annex $\mathrm{H}$ that applies to small wind turbines. In these summary results, wind speed is normalized to sealevel air density.

15. SUBJECT TERMS

ARE442 wind turbine; power performance; small wind turbine

\begin{tabular}{|c|c|c|}
\hline $\begin{array}{l}\text { a. REPORT } \\
\text { Unclassified }\end{array}$ & $\begin{array}{l}\text { b. ABSTRACT } \\
\text { Unclassified }\end{array}$ & $\begin{array}{l}\text { c. THIS PAGE } \\
\text { Unclassified }\end{array}$ \\
\hline
\end{tabular}

\begin{tabular}{|c|c|}
\hline $\begin{array}{l}\text { 17. LIMITATION } \\
\text { OF ABSTRACT }\end{array}$ & $\begin{array}{l}\text { 18. NUMBER } \\
\text { OF PAGES }\end{array}$ \\
\hline UL & \\
\hline
\end{tabular}

19a. NAME OF RESPONSIBLE PERSON

19b. TELEPHONE NUMBER (Include area code) 